\title{
FAITHFULLY FLAT DESCENT OF ALMOST PERFECT COMPLEXES IN RIGID GEOMETRY
}

\author{
AKHIL MATHEW
}

\begin{abstract}
We prove a version of faithfully flat descent in rigid analytic geometry, for almost perfect complexes and without finiteness assumptions on the rings involved. This extends results of Drinfeld for vector bundles.
\end{abstract}

\section{INTRODUCTION}

We begin with the statement of faithfully flat descent in rigid analytic geometry. Let $K$ be a complete nonarchimedean field, and let $A \rightarrow A^{\prime}$ be a faithfully flat map of $K$-affinoid algebras. In ordinary algebra, faithfully flat descent [2, Exp. VIII] states that the category of $A$-modules can be described as the category of $A^{\prime}$-modules with descent data, which take place over the tensor products $A^{\prime} \otimes_{A} A^{\prime}, A^{\prime} \otimes_{A} A^{\prime} \otimes_{A} A^{\prime}$. In rigid geometry, one obtains a similar conclusion, but with the tensor products replaced with completed tensor products and only for finitely generated modules.

Given a $K$-affinoid algebra $B$, we recall that $B$ is noetherian, and we let $\operatorname{Coh}(B)$ denote the category of finitely generated $B$-modules. One has the following result, due to Bosch-Görtz [13; the discretely valued case was previously known to Gabber (cf. [34, Theorem 1.9]). See also [15] and [3, Sec. 5.11] for accounts.

Theorem 1.1 (Bosch-Görtz-Gabber). Let $A \rightarrow A^{\prime}$ be a faithfully flat map of K-affinoid algebras. We have an equivalence of categories

$$
\operatorname{Coh}(A) \simeq \lim _{\longleftarrow}\left(\operatorname{Coh}\left(A^{\prime}\right) \rightrightarrows \operatorname{Coh}\left(A^{\prime} \hat{\otimes}_{A} A^{\prime}\right) \underset{\rightarrow}{\rightarrow} \cdots\right) .
$$

In other words, to specify a finitely generated $A$-module is equivalent to specifying a finitely generated $A^{\prime}$-module with descent data over the completed tensor products $A^{\prime} \hat{\otimes}_{A} A^{\prime}, A^{\prime} \hat{\otimes}_{A} A^{\prime} \hat{\otimes}_{A} A^{\prime}$.

There are at least two ways in which one could hope to generalize Theorem 1.1 The first is to work with the derived $\infty$-category; in the algebraic setting, one can generalize faithfully flat descent to derived $\infty$-categories [31, Sec. D.6.3]. In the rigid analytic case, one imposes the following finiteness condition, introduced in [1, Exp. I].

Definition 1.2 (Almost perfect complexes). For a ring $R$, we consider the derived $\infty$-category $D(R)$. An object $M \in D(R)$ is called almost perfect (or pseudocoherent) if it can be represented (up to quasi-isomorphism) by a chain complex $M_{\bullet}$ such that $M_{i}=0$ for $i \ll 0$ and each $M_{i}$ is finitely generated projective. We let $\operatorname{APerf}(R) \subset D(R)$ be the full subcategory spanned by almost perfect objects.

Example 1.3. Suppose $R$ is noetherian. Then $M \in D(R)$ is almost perfect if and only if $H_{i}(M)$ is finitely generated for each $i$ and vanishes for $i \ll 0$. In particular, $\operatorname{APerf}(R)$ is the (homologically) bounded-below derived $\infty$-category of the abelian category of finitely generated $R$-modules. 
From Theorem 1.1 and in view of Example 1.3. one may deduce the following extension of faithfully flat descent to almost perfect complexes; compare Hennion-Porta-Vezzosi [25, Sec. 3] for closely related results.

Theorem 1.4. The construction $A \mapsto \operatorname{APerf}(A)$ satisfies flat hyperdescent on $K$-affinoid algebras 1 In particular, if $A \rightarrow A^{\prime}$ is a faithfully flat map of $K$-affinoid algebras, then

$$
\operatorname{APerf}(A) \stackrel{\sim}{\longrightarrow} \lim _{\longleftarrow}\left(\operatorname{APerf}\left(A^{\prime}\right) \rightrightarrows \operatorname{APerf}\left(A^{\prime} \hat{\otimes}_{K} A^{\prime}\right) \underset{\rightarrow}{\rightarrow} \ldots\right) .
$$

The second way one may attempt to generalize Theorem 1.1 is to allow more general rings than $K$-affinoid algebras. The context of rigid geometry imposes strong finiteness assumptions: in particular, the rings $A, A^{\prime}$ are noetherian. A result of Drinfeld shows that descent for vector bundles (rather than finitely generated modules) holds very generally. We next formulate a version of Drinfeld's theorem.

Definition 1.5 (The site $\operatorname{Alg}_{\mathcal{O}_{K}}^{b}$ ). Let $\mathcal{O}_{K} \subset K$ be the ring of integers, and let $\pi \in \mathcal{O}_{K}$ denote a nonzero nonunit. Let $\operatorname{Alg}_{\mathcal{O}_{K}}^{b}$ denote the category of $\mathcal{O}_{K}$-algebras $R$ which are $\pi$-torsion-free and $\pi$-adically complete. We say that a map $R \rightarrow R^{\prime}$ in $\operatorname{Alg}_{\mathcal{O}_{K}}^{b}$ is $\pi$-completely faithfully flat if $R / \pi \rightarrow R^{\prime} / \pi$ is faithfully flat; this defines the $\pi$-completely flat topology on $\left(\operatorname{Alg}_{\mathcal{O}_{K}}^{b}\right)^{o p}$.

For any $\operatorname{ring} A$, we let $\operatorname{Vect}(A)$ denote the category of finitely generated projective $A$-modules. Then one has the following result. Compare [16, Th. 3.11] and [17, Prop. 3.5.4].

Theorem 1.6 (Drinfeld). The construction $R \mapsto \operatorname{Vect}(R[1 / \pi])$ is a sheaf of categories on $\operatorname{Alg}_{\mathcal{O}_{K}}^{b}$. That is, given $R \rightarrow R^{\prime}$ in $\operatorname{Alg}_{\mathcal{O}_{K}}^{b}$ which is $\pi$-completely faithfully flat, the natural functor

$$
\operatorname{Vect}(R[1 / \pi]) \rightarrow \underset{\lim }{\longleftarrow}\left(\operatorname{Vect}\left(R^{\prime}[1 / \pi]\right) \rightrightarrows \operatorname{Vect}\left(\widehat{R^{\prime} \otimes_{R} R^{\prime}}[1 / \pi]\right) \stackrel{\rightarrow}{\rightarrow} \ldots\right)
$$

is an equivalence of categories.

In this note, we will prove various common generalizations of Theorem [1.6 and Theorem 1.4 using some simplifications that occur when one works in the derived context. An instance of the result is the following.

Theorem 1.7. The construction $R \mapsto \operatorname{APerf}(R[1 / \pi])$ is a hypercomplete sheaf of $\infty$-categories on $\mathrm{Alg}_{\mathcal{O}_{K}}^{b}$. In particular, given $R \rightarrow R^{\prime}$ in $\operatorname{Alg}_{\mathcal{O}_{K}}^{b}$ which is $\pi$-completely faithfully flat, the natural functor induces an equivalence

$$
\operatorname{APerf}(R[1 / \pi]) \simeq \lim _{\longleftarrow}\left(\operatorname{APerf}\left(R^{\prime}[1 / \pi]\right) \rightrightarrows \operatorname{APerf}\left(\widehat{R^{\prime} \widehat{\otimes_{R}} R^{\prime}}[1 / \pi]\right) \stackrel{\rightarrow}{\rightarrow} \cdots\right) .
$$

This remains true if we replace APerf with $\mathrm{APerf}_{\geq 0} \subset \mathrm{APerf}$ (the subcategory of connective objects) or Perf $\subset$ APerf (the subcategory of perfect complexes), or the subcategories Perf $[a, b] \subset$ Perf of perfect complexes with Tor-amplitude in $[a, b]$.

The passage from Theorem[1.1 to Theorem 1.4 is facilitated by the $t$-structure on APerf in the $K$ affinoid case. By contrast, in the setting of Theorem [1.7 there is no $t$-structure on $\operatorname{APerf}(R[1 / \pi])$. As a consequence, we do not know how to deduce Theorem 1.7 from Theorem 1.6, and we will give a different argument: we will construct a stable $\infty$-category $\mathcal{M}(R)$ into which $\operatorname{APerf}(R[1 / \pi])$ embeds, and such that $\mathcal{M}(R)$ admits a $t$-structure that enables one to prove descent results. Our

\footnotetext{
${ }^{1}$ One also has descent for hypercovers instead only for Čech covers; this is additional information when one works with $\infty$-categories.
} 
main ingredients are the monadicity theorem of Barr-Beck-Lurie and a tool that exists only in the derived sense: the equivalence between torsion and complete modules [19, Th. 2.1]. Although it will not strictly be necessary to the proof, our argument is inspired by a result of Bhatt [7 that in this case states that any $\pi$-complete $\mathcal{O}_{K}$-module $M$ such that $M[1 / \pi]=0$ is in fact annihilated by a fixed power of $\pi$.

In fact, we will prove two versions of our descent result (Theorem 7.8 and Theorem 5.8 below). The first is a generalization of Theorem 1.7 to the case of a finitely generated ideal (rather than simply inverting an element). The second is one where $\pi$-complete faithful flatness is replaced by universal descent in the sense of [32, Sec. 3], which for finitely presented maps is equivalent to being a $v$-cover 37 .

Part of the difficulty in proving descent results such as the above is the lack of a well-behaved theory of quasicoherent (rather than coherent) sheaves in analytic (e.g., rigid) geometry. Recently Clausen and Scholze have, using condensed mathematics, defined a category of quasicoherent sheaves on an extremely large class of analytic spaces 38 . We expect that our results can be obtained using their framework. Compare also recent work of Andreychev 4, which uses condensed mathematics to prove some related descent statements (for the analytic rather than flat topology).

Conventions. We will generally use the notations and conventions of [30, 31, and formulate our results for connective $E_{\infty}$-rings rather than for ordinary commutative rings. In many cases this is essential even for questions that begin with discrete rings, because the derived completion process may introduce higher homotopy groups. In particular, all tensor products will be derived tensor products.

Given a connective $E_{\infty}$-ring $R$, we write $\operatorname{Mod}(R)$ for the $\infty$-category of $R$-module spectra. When $R$ is an ordinary commutative ring, this recovers the unbounded derived $\infty$-category $D(R)$. The stable $\infty$-category $\operatorname{Mod}(R)$ is equipped with a $t$-structure, and we let $\operatorname{Mod}(R)_{\geq 0}, \operatorname{Mod}(R)_{\leq 0} \subset$ $\operatorname{Mod}(R)$ denote the subcategories of connective and coconnective objects. We let $\operatorname{Mod}(R)^{\complement}$ denote the (usual) abelian category of discrete $\pi_{0}(R)$-modules, which is the heart of this $t$-structure. We will use homological indexing conventions.

We will freely use the theory of $t$-structures on stable $\infty$-categories as in [30, Sec. 1.2.1] (after [6] for triangulated categories). Given a stable $\infty$-category $\mathcal{C}$ equipped with a $t$-structure, we say that $\mathcal{C}$ is right-bounded if $\mathcal{C}=\bigcup_{n} \mathcal{C}_{\geq n}$ and left-complete if $\mathcal{C} \simeq \lim _{n} \mathcal{C}_{\leq n}$ (where the transition maps are the truncation maps).

Acknowledgments. I would like to thank Benjamin Antieau, Bhargav Bhatt, Dustin Clausen, Brian Conrad, Adriano Córdova, Vladimir Drinfeld, Ofer Gabber, Aron Heleodoro, Arthur-César Le Bras, Jacob Lurie, Peter Scholze, and the referee for helpful discussions and comments, and the Institute for Advanced Study for hospitality. This work was done while the author was a Clay Research Fellow.

\section{ISOGENIES}

2.1. Generalities. Throughout, we fix a connective $E_{\infty}$-ring $R$ (e.g., a discrete ring $R$ ) and an ideal $I \subset \pi_{0}(R)$. Let $\mathcal{C}$ be an $R$-linear additive $\infty$-category. Most often, we will take $\mathcal{C}$ to be a subcategory of the $\infty$-category $\operatorname{Mod}(R)$ of $R$-modules. Our basic "meta-definition" is the following.

Definition 2.1 (Properties up to isogeny). Let $\mathcal{P} \subset \mathcal{C}$ be a full subcategory stable under finite direct sums and retracts. We define a full subcategory $\mathcal{P}_{\leq I} \subset \mathcal{C}$ as follows: an object $X \in \mathcal{C}$ belongs to $\mathcal{P}_{\leq I}$ if for each $a \in I$, there exists $X_{0} \in \mathcal{P}$ (depending on $a$ ) and maps $f: X \rightarrow X_{0}, g: X_{0} \rightarrow X$ 
with $g \circ f: X \rightarrow X$ given by multiplication by $a$. We also write $\mathcal{P}_{\leq I^{\infty}}=\bigcup_{n>0} \mathcal{P}_{\leq I^{n}}$; informally, we can think of $\mathcal{P}_{\leq I}$ as those objects which satisfy the defining property of objects in $\mathcal{P}$ "up to isogeny."

In this note, we will be interested almost exclusively in the case where $I \subset \pi_{0}(R)$ is a finitely generated ideal. By contrast, when $I=I^{2}$, this type of definition is frequently used in almost ring theory [23].

Remark 2.2. (1) When $I=(1)$, then $\mathcal{P}_{\leq I}=\mathcal{P}$. This follows because $\mathcal{P}$ is closed under retracts.

(2) For convenience, we have formulated the above for $\infty$-categories, but the above definition only depends on the underlying homotopy category $\operatorname{Ho}(\mathcal{C})$ (which is naturally enriched in $\pi_{0}(R)$-modules).

(3) Let $F: \mathcal{C} \rightarrow \mathcal{D}$ be an $R$-linear functor of $R$-linear additive $\infty$-categories. Suppose $\mathcal{P} \subset$ $\mathcal{C}, \mathcal{P}^{\prime} \subset \mathcal{D}$ are full subcategories closed under finite direct sums and retracts. Suppose $F$ carries $\mathcal{P}$ into $\mathcal{P}^{\prime}$. Then $F$ carries $\mathcal{P}_{\leq I}$ into $\mathcal{P}_{\leq I}^{\prime}$.

(4) Let $J \subset \pi_{0}(R)$ be another ideal. Then $\left(\mathcal{P}_{\leq I}\right)_{\leq J} \subset \mathcal{P}_{\leq I J}$.

(5) Let $\left\{I_{\alpha}, \alpha \in A\right\}$ by a filtered system of ideals and let $I=\bigcup I_{\alpha}$. Then $\mathcal{P}_{\leq I}=\bigcap \mathcal{P}_{\leq I_{\alpha}}$.

It will be convenient to rephrase Definition 2.1 in the finitely generated case in terms of the notion of $\leq I$-split surjection (resp. injection), as will follow from Proposition 2.5 below.

Definition 2.3 ( $\leq I$-split surjections and injections). $\quad$ (1) A map of (discrete) $\pi_{0}(R)$-modules $M \rightarrow N$ is said to be $\leq I$-surjective if its cokernel is annihilated by $I$.

(2) A map $f: X \rightarrow Y$ in $\mathcal{C}$ is said to be a $\leq I$-split surjection if for all $Z \in \mathcal{C}$, the map of $\pi_{0}(R)$-modules $\pi_{0} \operatorname{Hom}_{\mathcal{C}}(Z, X) \rightarrow \pi_{0} \operatorname{Hom}_{\mathcal{C}}(Z, Y)$ is $\leq I$-surjective. This is equivalent to the statement that for each $a \in I$, there exists $g_{a}: Y \rightarrow X$ such that $f \circ g_{a}: Y \rightarrow Y$ is given by multiplication by $a$.

(3) A map $f: X \rightarrow Y$ in $\mathcal{C}$ is said to be a $\leq I$-split injection if for all $Z \in \mathcal{C}$, the map of $\pi_{0}(R)$-modules $\pi_{0} \operatorname{Hom}_{\mathcal{C}}(Y, Z) \rightarrow \pi_{0} \operatorname{Hom}_{\mathcal{C}}(X, Z)$ is $\leq I$-surjective. This is equivalent to the statement that for each $a \in I$, there exists $g_{a}: Y \rightarrow X$ such that $g_{a} \circ f: X \rightarrow X$ is given by multiplication by $a$.

Remark 2.4. Let $J \subset \pi_{0}(R)$ be another finitely generated ideal. The composite of a $\leq I$-split surjective (resp. $\leq I$-split injective) map and a $\leq J$-split surjective map (resp. $\leq J$-split injective map) is $\leq I J$-split surjective (resp. $\leq I J$-split injective).

Proposition 2.5. Suppose $I \subset \pi_{0}(R)$ is a finitely generated ideal. Then the following are equivalent:

(1) An object $X \in \mathcal{C}$ belongs to $\mathcal{P}_{\leq I}$.

(2) There exists $a \leq I$-split surjection $X^{\prime} \rightarrow X$ with $X^{\prime} \in \mathcal{P}$.

(3) There exists $a \leq I$-split injection $X \rightarrow X^{\prime \prime}$ with $X^{\prime \prime} \in \mathcal{P}$.

Proof. It is easy to see from the definitions that (2) or (3) implies (1). Suppose (1). Let $x_{1}, \ldots, x_{n} \in$ $I$ be a set of generators. We have objects $X_{1}^{\prime}, \ldots, X_{n}^{\prime} \in \mathcal{P}$ and maps $f_{i}: X \rightarrow X_{i}^{\prime}$ and $g_{i}: X_{i}^{\prime} \rightarrow X$ such that $g_{i} \circ f_{i}$ is multiplication by $x_{i}$. We can consider the map $\oplus_{i} f_{i}: X \rightarrow \bigoplus_{i=1}^{n} X_{i}^{\prime}$. Since the $x_{i}$ generate $I$, it is not difficult to see that this map is a $\leq I$-split injection. Similarly, the map $\oplus_{i} g_{i}: \bigoplus_{i=1}^{n} X_{i}^{\prime} \rightarrow X$ is a $\leq I$-split surjection.

Corollary 2.6. Suppose $I$ is finitely generated and $\mathcal{P}$ is the filtered union of the full additive, idempotent-complete subcategories $\mathcal{P}_{\alpha}, \alpha \in A$. Then $\mathcal{P}_{\leq I}=\bigcup_{\alpha \in A}\left(\mathcal{P}_{\alpha}\right)_{\leq I}$. 
We will use Definition 2.1 in the following instances.

Definition 2.7. (1) Suppose $\mathcal{P}$ is the subcategory of zero objects. Then an object $X \in \mathcal{C}$ belongs to $\mathcal{P}_{\leq I}$ if and only if every element of $I$ acts by zero on $X$. In this case, we will say that $X$ is $\leq \bar{I}$-isogenous to zero.

(2) Let $\mathcal{D}=\operatorname{Fun}\left(\Delta^{1}, \mathcal{C}\right)$ denote the $\infty$-category of arrows $X \rightarrow X^{\prime}$ in $\mathcal{C}$, and let $\mathcal{P}$ be the subcategory of isomorphisms. An arrow in $\mathcal{C}$ belonging to $\mathcal{P} \leq I$ is said to be an $\leq I$-isogeny.

(3) Suppose $\mathcal{C}$ is a compactly generated $R$-linear additive $\infty$-category and $\mathcal{P}$ is the subcategory of compact objects. We say that an object is $\leq I$-compact if it belongs to $\mathcal{P}_{\leq I}$.

For the next results, we note that there is a small subtlety that the construction of the arrow $\infty$ category does not commute with taking homotopy categories, i.e., $\operatorname{Ho}\left(\operatorname{Fun}\left(\Delta^{1}, \mathcal{C}\right)\right) \neq \operatorname{Fun}\left(\Delta^{1}, \operatorname{Ho}(\mathcal{C})\right)$ (if so, the proofs could be shortened). Instead, we have a fiber sequence

$$
\operatorname{Hom}_{\mathrm{Fun}\left(\Delta^{1}, \mathcal{C}\right)}\left(X \rightarrow Y, X^{\prime} \rightarrow Y^{\prime}\right) \rightarrow \operatorname{Hom}_{\mathcal{C}}\left(X, X^{\prime}\right) \times \operatorname{Hom}_{\mathcal{C}}\left(Y, Y^{\prime}\right) \rightarrow \operatorname{Hom}_{\mathcal{C}}\left(X, Y^{\prime}\right)
$$

Proposition 2.8 (Characterization of $\leq I$-isogenies). If an arrow $f: X \rightarrow Y$ is $a \leq I$-isogeny, then for each $a \in I$, there exists $g_{a}: Y \rightarrow X$ such that $f \circ g_{a}$ and $g_{a} \circ f$ are given by multiplication by a. Conversely, if this condition holds, then $f$ is $a \leq I^{2}$-isogeny.

Proof. The first direction is a diagram-chase, which we leave to the reader. For the other direction, suppose there exist maps $g_{a}$ as in the statement. Consider the natural morphism in $\operatorname{Fun}\left(\Delta^{1}, \mathcal{C}\right)$ given by $(X \stackrel{f}{\rightarrow} Y) \rightarrow(Y \stackrel{\text { id }}{\rightarrow} Y)$. One sees from (11) that this map is a $\leq I^{2}$-split injection, which shows that $f$ is a $\leq I^{2}$-isogeny.

Remark 2.9. Suppose $\mathcal{C}$ is actually a 1-category. Suppose $f: X \rightarrow Y$ is an arrow in $\mathcal{C}$ such that there exist maps $g_{a}: Y \rightarrow X$ for $a \in I$ as in Proposition 2.8. Then $f$ is a $\leq I$-isogeny; indeed, for each $a$, we consider the diagram

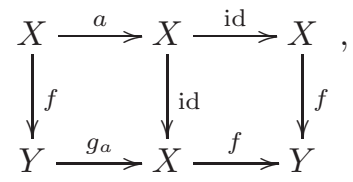

such that the composite map is multiplication by $a$ in the arrow category. In particular, if $\mathcal{C}$ is a 1-category, then Proposition 2.8 simplifies to an if and only if assertion.

We will need a variant of the five-lemma, whose proof we leave to the reader.

Lemma 2.10. Let

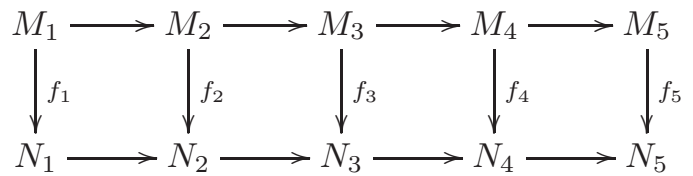

be a commutative diagram of discrete $\pi_{0}(R)$-modules with exact rows. Suppose $f_{2}, f_{5}$ are isomorphisms and $f_{4}$ is $\leq I$-surjective. Then $f_{3}$ is $\leq I$-surjective.

Proposition 2.11. Let $f=\left(f_{1}, f_{2}\right):(X \rightarrow Y) \rightarrow\left(X^{\prime} \rightarrow Y^{\prime}\right)$ be a map in $\operatorname{Fun}\left(\Delta^{1}, \mathcal{C}\right)$, leading to a commutative square

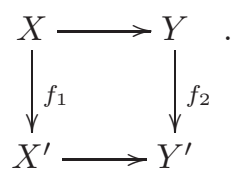


Suppose $f_{1}: X \rightarrow X^{\prime}$ is $\leq I$-split surjective and $f_{2}$ is an equivalence. Then $f$ is $\leq I$-split surjective. Similarly, suppose $f_{1}$ is an equivalence and $f_{2}$ is $\leq I$-split injective. Then $f$ is $\leq I$-split injective.

Proof. Combine Lemma 2.10 and the fiber sequence (1).

Proposition 2.12. Let $\mathcal{P}, \mathcal{Q} \subset \mathcal{C}$ and suppose $I, J \subset \pi_{0}(R)$ be ideals. Let $\operatorname{Fun}\left(\Delta^{1}, \mathcal{C}\right)$ be the $\infty$ category of arrows in $\mathcal{C}$. Let $\mathcal{R} \subset \operatorname{Fun}\left(\Delta^{1}, \mathcal{C}\right)$ be the subcategory of arrows $X \rightarrow Y$ with $X \in \mathcal{P}, Y \in$ $\mathcal{Q}$. Then an arrow $X^{\prime} \rightarrow Y^{\prime}$ in $\operatorname{Fun}\left(\Delta^{1}, \mathcal{C}\right)$ with $X^{\prime} \in \mathcal{P}_{\leq I}$ and $Y^{\prime} \in \mathcal{Q}_{\leq J}$ belongs to $\mathcal{R}_{\leq I J}$.

Proof. Without loss of generality, we can assume $I, J$ are finitely generated. By assumption and Proposition 2.5. there is a $\leq I$-split surjection $W^{\prime} \rightarrow X^{\prime}$ with $W^{\prime} \in \mathcal{P}$. We then get a map of arrows $\left(W^{\prime} \rightarrow Y^{\prime}\right) \rightarrow\left(X^{\prime} \rightarrow Y^{\prime}\right)$ which is necessarily a $\leq I$-split surjection in $\operatorname{Fun}\left(\Delta^{1}, \mathcal{C}\right)$ by Proposition 2.11. Therefore, it suffices to show that $\left(W^{\prime} \rightarrow Y^{\prime}\right) \in \mathcal{R}_{\leq J}$. But (by Proposition 2.5 again) we have a $\leq J$-split injection $Y^{\prime} \rightarrow Z^{\prime}$ with $Z^{\prime} \in \mathcal{P}$. We get a map $\left(W^{\prime} \rightarrow Y^{\prime}\right) \rightarrow\left(W^{\prime} \rightarrow Z^{\prime}\right)$ which is a $\leq J$-split injection via Proposition 2.11 However, $\left(W^{\prime} \rightarrow Z^{\prime}\right) \in \mathcal{R}$, so we conclude.

Our main application of Proposition 2.12 is that the $\leq I$-construction behaves well with respect to extensions.

Corollary 2.13. Suppose $\mathcal{C}$ is an R-linear stable $\infty$-category, and let $I, J \subset \pi_{0}(R)$ be finitely generated ideals. Let $\mathcal{P}, \mathcal{Q}, \mathcal{R}$ be full additive, idempotent-complete subcategories. Suppose the cofiber of any map with source in $\mathcal{P}$ and target in $\mathcal{Q}$ belongs to $\mathcal{R}$. Then the cofiber of any map with source in $\mathcal{P}_{\leq I}$ and target in $\mathcal{Q}_{\leq J}$ belongs to $\mathcal{R}_{\leq I J}$.

Proof. This is a consequence of Proposition 2.12, We consider the cofiber functor cofib: $\operatorname{Fun}\left(\Delta^{1}, \mathcal{C}\right) \rightarrow$ $\mathcal{C}$. Let $\mathcal{W} \subset \operatorname{Fun}\left(\Delta^{1}, \mathcal{C}\right)$ be the subcategory of arrows with source in $\mathcal{P}$ and target in $\mathcal{Q}$. The cofiber functor carries $\mathcal{W}$ into $\mathcal{R}$ and therefore $\mathcal{W}_{\leq I J}$ into $\mathcal{R}_{\leq I J}$. By Proposition 2.12, any arrow with source in $\mathcal{P}_{\leq I}$ and target in $\mathcal{Q}_{\leq J}$ belongs to $\mathcal{W}_{\leq I J}$.

Corollary 2.14. Suppose $\mathcal{C}$ is an $R$-linear stable $\infty$-category and $\mathcal{P} \subset \mathcal{C}$ a thick subcategory. Then $\mathcal{P}_{\leq I \infty} \subset \mathcal{C}$ is also a thick subcategory.

Proposition 2.15 (Characterization of $\leq I$-compact objects). Let $\mathcal{C}$ be a compactly generated $R$-linear additive $\infty$-category. Then the following are equivalent for $X \in \mathcal{C}$ :

a) $X$ is $\leq I$-compact.

b) For every filtered system $Y_{i}, i \in \mathcal{I}$ in $\mathcal{C}$, the map

$$
\underset{\lim }{\longrightarrow} \pi_{0} \operatorname{Hom}_{\mathcal{C}}\left(X, Y_{i}\right) \rightarrow \pi_{0} \operatorname{Hom}_{R}\left(X, \lim _{\longrightarrow} Y_{i}\right)
$$

is an $\leq I$-isogeny of $\pi_{0} R$-modules.

c) For every filtered system $Y_{i}, i \in \mathcal{I}$ in $\mathcal{C}$, the map

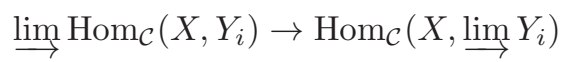

is an $\leq I$-isogeny of $R$-modules.

Proof. Suppose a). For every filtered system $Y_{i}, i \in I$, we define a functor on $\mathcal{C}$ with values in arrows in $R$-modules, given by $M^{\prime} \mapsto\left(\lim _{\mathfrak{L}} \operatorname{Hom}_{\mathcal{C}}\left(X, Y_{i}\right) \rightarrow \operatorname{Hom}_{\mathcal{C}}\left(X, \underline{\lim } Y_{i}\right)\right)$. This carries compact

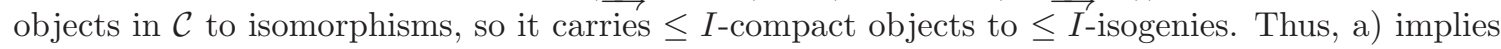
c), and c) clearly implies b). To see that b) implies a), we write $X$ as a filtered colimit of compact objects and use that (2) is a $\leq I$-isogeny to exhibit $X$ as $\leq I$-compact. 
2.2. Modules. We now specialize to the case where $\mathcal{C}$ is given by modules over a connective $E_{\infty^{-}}$ ring $R$ (or some appropriate subcategory), and begin by reviewing some finiteness conditions.

Definition 2.16 ( $\leq I$-finitely generated modules). Suppose $\mathcal{C}=\operatorname{Mod}^{\mathcal{P}}(R)$ is the category of discrete $R$-modules, and $\mathcal{P}$ is the subcategory of finitely generated modules. Then we say that a discrete $R$-module $M$ is $\leq I$-finitely generated if it belongs to $\mathcal{P}_{\leq I}$. It is not difficult to see that this holds if and only if $M$ is $\leq I$-isogenous to a finitely generated module.

Definition 2.17 (Perfect and almost perfect modules). Let $\operatorname{Perf}(R) \subset \operatorname{Mod}(R)$ denote the $\infty$ category of perfect $R$-modules, or equivalently the compact objects in $\operatorname{Mod}(R)$. We let $\operatorname{APerf}(R) \subset$ $\operatorname{Mod}(R)$ denote the subcategory of almost perfect (or pseudocoherent) $R$-modules (see [30, Sec. 7.2.4]; in the discrete case the definition is due to [1, Exp. I]). Given a qcqs spectral scheme $X$, we let $\mathrm{QCoh}(X)$ denote the $\infty$-category of quasi-coherent modules on $X$, and $\operatorname{Perf}(X), \operatorname{APerf}(X) \subset$ $\mathrm{QCoh}(X)$ the associated subcategories of perfect and almost perfect objects.

Our goal is to study $\leq I^{\infty}$-versions of perfectness and almost perfectness. For this, we will need to use the following intermediate property. We review the notion of being "perfect to order $n$," as in [31, Sec. 2.7] or [40, Tag 064N], and then formulate its $\leq I$-analog.

Definition 2.18. We say that a bounded-below object $M \in \operatorname{Mod}(R)$ is perfect to order $n$ if the following equivalent (by [31, Prop. 2.7.0.4]) conditions are satisfied:

(1) For every filtered system $N_{i}, i \in \mathcal{I}$ in $\operatorname{Mod}(R)_{\leq n}$, the natural map of $R$-module spectra

$$
\underset{\lim }{\longrightarrow} \operatorname{Hom}_{R}\left(M, N_{i}\right) \rightarrow \operatorname{Hom}_{R}\left(M, \underset{\lim }{\longrightarrow} N_{i}\right)
$$

has homotopy fiber in $\operatorname{Mod}(R)_{<0}$ : that is, it induces an isomorphism on $\pi_{i}$ for $i>0$ and an injection on $\pi_{0}$.

(2) For every filtered system $N_{i}, i \in \mathcal{I}$ in $\operatorname{Mod}(R)_{\leq n}$ such that each transition map $N_{i} \rightarrow N_{j}$ induces an injection on $\pi_{n}$, the natural map (4) is an isomorphism on connective covers.

(3) For every filtered system $N_{i}, i \in \mathcal{I}$ of discrete $\pi_{0}(R)$-modules, the natural map

$$
\underset{\lim }{\longrightarrow} \operatorname{Ext}_{R}^{i}\left(M, N_{i}\right) \rightarrow \operatorname{Ext}_{R}^{i}\left(M, \underset{\lim }{\longrightarrow} N_{i}\right)
$$

is an isomorphism for $i<n$ and an injection for $i=n$.

The condition of being perfect to order $n$ only depends on the truncation $\tau_{\leq n} M \in \operatorname{Mod}(R)_{\leq n}$, so we will often view being "perfect to order $n$ " as a property of objects in $\operatorname{Mod}(R)_{\leq n}$. Note finally that a bounded-below $R$-module $M$ is almost perfect if and only if it is perfect to each order $n$.

Example 2.19. An object $M \in \operatorname{Mod}(R)_{\geq 0}$ is perfect to order zero if and only if $\pi_{0}(M)$ is a finitely generated $\pi_{0}(R)$-module.

Example 2.20. Let $M$ be a compact object of $\operatorname{Mod}(R)_{\leq n}$ (so $\tau_{\leq n}$ of a perfect $R$-module). Choose a surjection of discrete $\pi_{0}(R)$-modules, $\pi_{n} M \rightarrow M_{n}^{\prime}$; then the pushout $M \sqcup_{\left(\pi_{n} M\right)[n]} M_{n}^{\prime}[n]$ is an example of an object in $\operatorname{Mod}(R)_{[0, n]}$ which is perfect to order $n$. This is straightforward to see using the above criteria. Conversely, any object of $\operatorname{Mod}(R)_{[0, n]}$ which is perfect to order $n$ arises in the above fashion. See [31, Cor. 2.7.2.2].

Proposition 2.21. Let $M^{\prime} \rightarrow M \rightarrow M^{\prime \prime}$ be a cofiber sequence of bounded-below R-modules. Suppose $M$ is perfect to order $n$. Then the following are equivalent:

(1) $M^{\prime}$ is perfect to order $n-1$.

(2) $M^{\prime \prime}$ is perfect to order $n$. 
Proof. This follows from a diagram-chase. Let $N_{i}, i \in I$ be a filtered system in $\operatorname{Mod}(R)_{[0, n]}$. Let $F$ be the functor which carries an $R$-module $Q$ to $\operatorname{fib}\left(\lim _{\operatorname{Hom}}\left(Q, N_{i}\right) \rightarrow \operatorname{Hom}_{R}\left(Q, \lim N_{i}\right)\right)$. By assumption, $F(M) \in \operatorname{Mod}(R)_{<0}$; since we have a fiber sequence $F\left(M^{\prime \prime}\right) \rightarrow F(M) \rightarrow \vec{F}\left(M^{\prime}\right)$, the result now follows easily.

Definition 2.22 (Modules $\leq I$-perfect to order $n$ ). Let $M \in \operatorname{Mod}(R)_{<n}$. If $\mathcal{P} \subset \operatorname{Mod}(R)_{<n}$ is the subcategory of objects which are perfect to order $n$, then we will say that $M$ is $\leq I$-perfect to order $n$ if $M$ belongs to $\mathcal{P}_{\leq I}$. Given an arbitrary $R$-module $M$, we will say that $M$ is $\leq I$-perfect to order $n$ if $\tau_{\leq n} M$ is. An object is $\leq I^{\infty}$-perfect to order $n$ if it is $\leq I^{r}$-perfect to order $n$, for some $r$.

Proposition 2.23. Let $M \in \operatorname{Mod}(R)$ be $\leq I^{\infty}$-perfect to order $n$. Let $N \in \operatorname{Mod}(R)_{\leq n-1}$. Then for $t \in I$, the map $\tau_{\geq 0} \operatorname{Hom}_{R}(M, N)[1 / t] \rightarrow \tau_{\geq 0} \operatorname{Hom}_{R}(M[1 / t], N[1 / t])$ is an isomorphism.

Proof. Note that this question depends only on $\tau_{\leq n-1} M$. Our assumption implies that $\tau_{\leq n-1} M$ receives a $\leq I^{\infty}$-split surjection from $\tau_{\leq n-1}$ of a perfect $R$-module $P$. Therefore, we find that $\tau_{\geq 0} \operatorname{Hom}_{R}(M, N)[1 / t]$ and $\tau_{\geq 0} \operatorname{Hom}_{R}(M[\overline{1} / t], N[1 / t])$ are naturally retracts of $\tau_{\geq 0} \operatorname{Hom}_{R}(P, N)[1 / t]$ and $\tau_{\geq 0} \operatorname{Hom}_{R}(P[1 / t], N[1 / t])$. Since $\tau_{\geq 0} \operatorname{Hom}_{R}(P, N)[1 / t] \rightarrow \tau_{\geq 0} \operatorname{Hom}_{R}(P[1 / t], N[1 / t])$ is an isomorphism, we obtain the result for $M$ as well.

Proposition 2.24. Let $M \in \operatorname{Mod}(R)_{\leq n}$ be bounded-below. In order for $M$ to be $\leq I$-perfect to order $n$, it is necessary and sufficient that for every filtered system $N_{i}, i \in \mathcal{I}$ in $\operatorname{Mod}(R)_{\leq n}$ with injective transition maps on $\pi_{n}$, the connective cover of the natural map (4) is an $\leq I$-isogeny.

Proof. Without loss of generality, we can assume $I$ finitely generated. Necessity is clear, since the connective cover of (4) is an equivalence for $M$ perfect to order $n$ under our assumptions. We can find a filtered system $N_{i} \in \operatorname{Mod}(R)_{\leq n}$ with injective transition maps on $\pi_{n}$ such that each $N_{i}$ is perfect to order $n$ and such that $\lim N_{i} \simeq M$, e.g., using Example 2.20 and that $M$ is a filtered colimit of perfect modules. Our assumption now shows that one of the $N_{i}$ maps to $M$ via a $\leq I$-split surjection, which is enough to imply the claim.

Example 2.25. An object $M \in \operatorname{Mod}(R)$ is $\leq I$-perfect to order zero if and only if $\pi_{0}(M)$ is $\leq I$-finitely generated.

Proposition 2.26. Let $M^{\prime} \rightarrow M \rightarrow M^{\prime \prime}$ be a cofiber sequence in $\operatorname{Mod}(R)$ with all terms boundedbelow. Suppose $M$ is perfect to order $n$. Then the following are equivalent:

(1) $M^{\prime}$ is $\leq I$-perfect to order $n-1$.

(2) $M^{\prime \prime}$ is $\leq I$-perfect to order $n$.

Proof. This follows from Proposition 2.21, in light of Corollary 2.13, Alternatively, one can argue directly using Proposition 2.24

2.3. Passage from the generic fiber. In this subsection, we will prove various instances of the following principle: under (derived) $I$-completeness assumptions, for a module to have a certain property outside $I$ is equivalent to having it up to $\leq I^{\infty}$-isogeny integrally, or equivalently up to $\leq I^{r}$ modulo $I^{n}$ for every $n$ (where $r$ is independent of $n$ ).

To begin with, we consider the case of discrete modules over a commutative ring. Fix a commutative ring $R$ and a finitely generated ideal $I \subset R$. Recall the notion of derived completeness; see [40, Tag 091N] or [31, Sec. 7.3] for accounts.

Definition 2.27 (Derived $I$-complete modules). A discrete $R$-module $M \in \operatorname{Mod}(R)^{\ominus}$ is derived $I$-complete if for each $x \in I$, we have $\lim ^{i}(\ldots \stackrel{x}{\rightarrow} M \stackrel{x}{\rightarrow} M)=0$ for $i=0,1$. The collection of derived 
$I$-complete modules forms an abelian subcategory of $\operatorname{Mod}(R)^{\ominus}$ which is closed under extensions, and which properly contains all classically $I$-complete modules. The inclusion of derived $I$-complete modules into all of $\operatorname{Mod}(R)^{\odot}$ admits a left adjoint given by derived $I$-completion (and truncation in degree zero), $M \mapsto \tau_{\leq 0}\left(\widehat{M}_{I}\right)$.

A derived $I$-complete module $M$ is generally not $I$-adically separated (i.e., one may have $\bigcap I^{n} M \neq$ $0)$; this is the key difference between derived $I$-complete and classically $I$-complete modules. Nonetheless, given a derived complete module $M \in \operatorname{Mod}(R)^{\odot}$, if $M / I M=0$ then $M=0$. In the future, we will need a slight refinement of this fact, as follows.

Lemma 2.28. Let $R$ be a commutative ring with a fixed element $t \in R$. Let $M, N, P$ be discrete $R$-modules and suppose $P$ is derived t-complete. Let $f: M \rightarrow N$ and $g: N \rightarrow P$ be maps. Suppose that both $f$ and $g$ are $t$-divisible elements of $\operatorname{Hom}_{R}(M, N), \operatorname{Hom}_{R}(N, P)$. Then $g \circ f=0$.

Proof. We will use the following basic fact: among $t$-torsion-free objects, derived and classical completion coincide; this follows, e.g., from the formula below (Construction 2.32). Clearly $g$ annihilates all $t$-power torsion elements of $N$, so it factors through a map $\left(\widehat{N / N_{\text {tors }}}\right) \rightarrow P$. It thus suffices to show that the composite map $M \stackrel{f}{\rightarrow} N \rightarrow\left(\widehat{N / N_{\text {tors }}}\right)$ is zero. Indeed, this composite map is divisible by arbitrary powers of $t$, but $\left(\widehat{N / N_{\text {tors }}}\right)$ (as the derived, and hence classical, $t$-completion of a $t$-torsion-free module) is $t$-adically separated, forcing the map to vanish.

Our starting point for this section is the following "uniform boundedness" result. See also [5. Lemma 2.2] for a special case (when $t=p$ ).

Theorem 2.29 (Bhatt [7). Let $R$ be a commutative ring containing a finitely generated ideal $I \subset R$. Let $M$ be a derived I-complete discrete $R$-module. Then the following are equivalent:

(1) $M$ vanishes away from $I$ : that is, $M[1 / t]=0$ for $t \in I$.

(2) There exists $r$ such that $M$ is $\leq I^{r}$-isogenous to zero.

(3) There exists $r$ such that for all $n, M / I^{n} M$ is $\leq I^{r}$-isogenous to zero.

Proof. The equivalence of (1) and (2) appears in [7. It remains only to show that (3) implies (2). Taking $n=r+1$, we find that $I^{r} M=I^{r+1} M=I\left(I^{r} M\right)$. Since $I^{r} M$ is derived $I$-adically complete (as the image of a map from a finite direct sum of copies of $M$ to $M$ ), it follows that $I^{r} M=0$, as desired.

Proposition 2.30. Let $R$ be a ring which is derived I-complete for some finitely generated ideal $I \subset R$. Let $M$ be a derived I-complete discrete $R$-module. Then the following are equivalent:

(1) $M$ is finitely generated outside $I$ (that is, for $t \in I, M[1 / t]$ is a finitely generated $R[1 / t]$ module).

(2) There exists $r$ such that $M$ is $\leq I^{r}$-finitely generated.

(3) There exists $r$ such that for all $n, M / I^{n} M$ is $\leq I^{r}$-finitely generated.

In fact, the best possible $r$ for (2) and (3) are the same.

Proof. Suppose (1). Then there exists a finitely generated free $R$-module $F$ and a map $f: F \rightarrow M$ which induces a surjection after inverting any $t \in I$ (it suffices to check this as $t$ runs over a system of generators). By Theorem [2.29, we have $I^{r} \operatorname{coker}(f)=0$ for some $r$. Replacing $F$ with $\operatorname{im}(f)$, we find that $M$ is $\leq I^{r}$-finitely generated, proving (2). Clearly (2) implies (1), so it suffices to show that (3) implies (2). Taking $n=r+1$, we have that $M / I^{r+1} M$ is $\leq I^{r}$-finitely generated. Therefore, there exists a finitely generated free $R$-module $F^{\prime}$ and a map $g: F^{\prime} \rightarrow M$ such that 
$\operatorname{coker}(g) / I^{r+1} \operatorname{coker}(g)$ is annihilated by $I^{r}$, and therefore $I^{r} \operatorname{coker}(g)=0$ by derived completeness. Thus $\operatorname{im}(g) \subset M$ is a $\leq I^{r}$-isogeny and the result follows.

Now we switch to the non-discrete setting: let $R$ be a connective $E_{\infty}$-ring, and let $I \subset \pi_{0}(R)$ be a finitely generated ideal. We briefly recall the theory of $I$-complete and $I$-torsion objects in $\operatorname{Mod}(R)$, from [19; see also [31, Sec. 7.3] for a detailed account.

Definition 2.31 (Complete and torsion modules). (1) Given $M \in \operatorname{Mod}(R), M$ is said to be $I$-torsion if all the homotopy groups are $I$-power torsion (i.e., every element in $\pi_{*}(M)$ is annihilated by a power of $I$ ).

(2) The $R$-module $M$ is said to be $I$-complete if for each $x \in I, \lim (\ldots \stackrel{x}{\rightarrow} M \stackrel{x}{\rightarrow} M)=0$ in $\operatorname{Mod}(R)$. This holds if and only if each homotopy group $\pi_{i}(M)$ is derived $I$-complete as a discrete $\pi_{0}(R)$-module.

(3) The inclusion of $I$-complete modules in $\operatorname{Mod}(R)$ admits a left adjoint, called $I$-completion.

Construction 2.32 (A formula for the completion). Suppose $I=\left(x_{1}, \ldots, x_{p}\right)$. For each $n>0$ and $1 \leq i \leq p$, let $R / / x_{i}=\operatorname{cofib}\left(x_{i}^{n}: R \rightarrow R\right)$ and $R_{n}=R / / x_{1} \otimes_{R} \cdots \otimes_{R} R / / x_{p}$. The family $\left\{R_{n}\right\}_{n \geq 1}$ forms a tower of perfect $R$-modules. Given any $M \in \operatorname{Mod}(R)$, the natural map $M \rightarrow \lim _{n}\left(M \otimes_{R} R_{n}\right)$ exhibits the target as the $I$-completion of $M$.

Note that this tower $\left\{R_{n}\right\}$ is not canonical (it relies on a choice of generators of $I$ ) and it does not generally form a tower of $E_{\infty}$-R-algebras, but only of $R$-modules. In the future, we will fix an explicit choice of generators of $I$ and thus of a tower $\left\{R_{n}\right\}$.

Throughout, we will need to use the following fundamental equivalence between complete and torsion modules, which goes back to [19, Th. 2.1] (see also [26, Th. 3.3.5]); cf. [31, Prop. 7.3.1.7] for an account in the present setting.

Theorem 2.33. Let $R$ be a connective $E_{\infty}$-ring, and let $I \subset R$ be a finitely generated ideal as above. The functor of I-completion also induces an equivalence between the subcategories of $I$ torsion modules and I-complete modules inside $\operatorname{Mod}(R)$.

Next, we prove the analogs of Proposition2.30 in the context of module spectra. The main results are similar: perfectness to any order on the generic fiber can be checked integrally (or modulo any power of the ideal in an appropriate sense) up to bounded isogeny.

Proposition 2.34. Let $R$ be a connective $E_{\infty}$-ring and $I=\left(x_{1}, \ldots, x_{p}\right) \subset \pi_{0}(R)$ a finitely generated ideal; fix a tower $\left\{R_{n}\right\}$ as in Construction 2.32. Let $M \in \operatorname{Mod}(R)$ be I-complete. Then the following are equivalent:

(1) For each $m<0, \pi_{m}(M)$ is $\leq I^{\infty}$-isogenous to zero.

(2) For each $m<0$, there exists $r$ such that $\pi_{m}\left(M \otimes_{R} R_{n}\right)$ is $\leq I^{r}$-isogenous to zero for all $n>0$.

(3) For each $m<0$ and $t \in I, \pi_{m}(M[1 / t])=0$.

Proof. It is easy to see that (1) implies (2) and (3). Furthermore, (3) implies (1) thanks to Theorem 2.29. It remains to show that (2) implies (1), so suppose (2). Since $M \simeq \lim _{n}\left(M \otimes_{R} R_{n}\right)$, we conclude via the Milnor exact sequence that each $\pi_{m}(M), m<-1$ is $\leq I^{\infty}$-isogenous to zero. It suffices to verify that $\pi_{-1}(M)$ is $\leq I^{\infty}$-isogenous to zero. Given what we have already shown, the hypotheses of the theorem are invariant under replacing $M$ with $\tau_{\geq-1} M$, so we may assume $M$ is $(-1)$-connective. Then we find that $\pi_{-1}\left(M \otimes_{R} R_{n}\right)=\pi_{-1}(M) /\left(x_{1}^{n}, \ldots, x_{p}^{n}\right) \pi_{-1}(M)$ is annihilated by a fixed power of $I$ uniformly in $n$, which forces (by Theorem 2.29) $\pi_{-1}(M)$ to be $\leq I^{\infty}$-isogenous to zero. 
Proposition 2.35. Let $R$ be a connective $E_{\infty}$-ring which is $I$-complete for $I=\left(x_{1}, \ldots, x_{p}\right) \subset \pi_{0}(R)$ a finitely generated ideal; fix $\left\{R_{n}\right\}$ as in Construction 2.32. Let $M$ be an object of $\operatorname{Mod}(R)$ which is bounded-below and I-complete. Given $m \geq 0$, the following are equivalent:

(1) For each $t \in I, M[1 / t] \in \operatorname{Mod}(R[1 / t])$ is perfect to order $m$.

(2) $M$ is $\leq I^{\infty}$-perfect to order $m$.

(3) There exists $r^{\prime}$ such that for all $n, M \otimes_{R} R_{n} \in \operatorname{Mod}(R)$ is $\leq I^{r^{\prime}}$-perfect to order $m$.

Proof. Without loss of generality (i.e., by shifting), we may assume $M$ connective. We use induction on $m$. In the case $m=0$, all three conditions are equivalent to $\pi_{0}(M)$ being $\leq I^{\infty}$-finitely generated (via Proposition 2.30). Now suppose $m>0$. Since all three conditions are isogeny invariant, we can assume without loss of generality that $\pi_{0}(M)$ is finitely generated. Choose a finitely generated free $R$-module $P$ and a map $P \rightarrow M$ inducing a surjection $\pi_{0}(P) \rightarrow \pi_{0}(M)$, and write $F=\operatorname{fib}(P \rightarrow M)$. Thanks to Proposition 2.26 conditions (1), (2), and (3) for $M$ are equivalent to the analogous conditions among (1), (2), and (3) for $F$ (with $m$ replaced by $m-1$ ). By induction on $m$, all of these three conditions are equivalent for $F$, and hence they are equivalent for $M$.

\section{The construction $\mathcal{M}(R)$}

Throughout this section, we fix a $E_{\infty}$-ring $R$ equipped with a finitely generated ideal $I \subset \pi_{0}(R)$. We will construct a stable $\infty$-category $\mathcal{M}(R)$, which we should regard as associated to the "generic fiber" of the formal spectrum $\operatorname{Spf}(R)$ (we will not need a precise notion here). Our main result is that $\operatorname{APerf}(\operatorname{Spec}(R) \backslash V(I))$ is naturally a full subcategory of $\mathcal{M}(R)$.

3.1. Definition of $\mathcal{M}(R)$. To begin with, we give the abelian version of the construction $\mathcal{M}(R)$; in fact, there are two natural candidates, involving complete and torsion modules respectively. In the derived setting, the distinction between the two goes away.

Definition 3.1 (The construction $\mathcal{A}(R)$ ). We let $\mathcal{A}(R)$ denote the abelian category obtained as the Serre quotient of the category of $I$-power torsion discrete $\pi_{0}(R)$-modules by the subcategory of those modules which are $\leq I^{\infty}$-isogenous to zero. Given a map $R \rightarrow R^{\prime}$, base-change gives a right exact functor $\mathcal{A}(R) \rightarrow \mathcal{A}\left(R^{\prime}\right)$ with a right adjoint (given by restriction of scalars) which is exact.

Definition 3.2 (The construction $\mathcal{B}(R)$ ). We let $\mathcal{B}(R)$ denote the abelian category obtained as the Serre quotient of the category of derived $I$-complete discrete $\pi_{0}(R)$-modules by the subcategory of those modules which are $\leq I^{\infty}$-isogenous to zero.

We can regard $\mathcal{B}(R)$ as an abelian version of the category of Banachian spaces studied in [17. Next, we need the stable versions.

Construction 3.3 ( $t$-structures on Verdier quotients). Let $\mathcal{E}$ be a stable $\infty$-category equipped with a $t$-structure with heart $\mathcal{E}^{\ominus}$. Given a Serre subcategory $\mathcal{B}_{0} \subset \mathcal{E}^{\ominus}$, we define a thick subcategory $\mathcal{E}^{\prime} \subset \mathcal{E}$ consisting of those objects all of whose homotopy groups lie in $\mathcal{B}_{0}$. Then $\mathcal{E}^{\prime}$ also inherits a $t$-structure, as does the Verdier quotient $\mathcal{E} / \mathcal{E}^{\prime}$. The heart $\mathcal{E}^{\prime}$ is given by $\mathcal{B}_{0}$ and the heart of of $\mathcal{E} / \mathcal{E}^{\prime}$ is given by the Serre quotient $\mathcal{E}^{\odot} / \mathcal{B}_{0}$.

Definition 3.4 (The constructions $\left.\mathcal{M}_{0}(R), \mathcal{M}(R)\right)$. $\quad(1)$ Let $\operatorname{Mod}(R)_{>-\infty}^{\mathrm{cpl}} \subset \operatorname{Mod}(R)$ denote the subcategory consisting of $R$-modules which are bounded-below and $I$-complete. Let $\operatorname{Mod}(R)_{>-\infty}^{\mathrm{nil}} \subset \operatorname{Mod}(R)_{>-\infty}^{\mathrm{cpl}}$ denote the thick subcategory spanned by those $M$ such that for each $i, \pi_{i}(M)$ is $\leq I^{\infty}$-isogenous to zero. A map with cofiber in $\operatorname{Mod}(R)_{>-\infty}^{\text {nil }}$ is said to be a quasi-isogeny. 
(2) We have an equivalence of $\infty$-categories between $\operatorname{Mod}(R)_{>-\infty}^{\mathrm{cpl}}$ and the $\infty$-category of $R$ modules which are bounded-below and $I$-torsion (Theorem 2.33 , since both functors involved have bounded amplitude). Therefore, $\operatorname{Mod}(R)_{>-\infty}^{\mathrm{cpl}}$ is equipped with a $t$-structure, which we refer to as the $I$-torsion $t$-structure, whose heart is the abelian category of $I$-power torsion discrete $\pi_{0}(R)$-modules. This restricts to a $t$-structure on $\operatorname{Mod}(R)_{>-\infty}^{\text {nil }}$ whose heart is the abelian category of $\pi_{0}(R)$-modules which are $\leq I^{\infty}$-isogenous to zero.

(3) We let $\mathcal{M}_{0}(R)$ denote the Verdier quotient $\operatorname{Mod}(R)_{>-\infty}^{\mathrm{cpl}} / \operatorname{Mod}(R)_{>-\infty}^{\text {nil }}$, as an $R$-linear stable $\infty$-category; it also inherits a right-bounded (i.e., every object is bounded below) $t$-structure whose heart is given by the abelian category $\mathcal{A}(R)$ (Construction 3.3). It is linear over $\operatorname{Perf}(R)$ and the action annihilates the $I$-torsion objects in $\operatorname{Perf}(R)$, so it becomes linear over $\operatorname{Perf}(\operatorname{Spec}(R) \backslash V(I))$.

We let $\mathcal{M}(R)$ denote its left completion [30, Sec. 1.2.1]. By construction, $\mathcal{M}(R) \simeq$ $\lim _{n}\left(\mathcal{M}_{0}(R)\right)_{\leq n}$ where the transition maps are the truncation functors. Similarly, $\mathcal{M}(R)$ is a stable $\infty$-category (linear over $\operatorname{Perf}(\operatorname{Spec}(R) \backslash V(I))$ ) with a $t$-structure, which we refer to as the $I$-torsion $t$-structure; for each $n$, we have $\mathcal{M}(R)_{\leq n}=\mathcal{M}_{0}(R)_{\leq n}$.

Remark 3.5. There is no distinction between the idempotent-complete and the non-idempotent complete Verdier quotient in the definition of $\mathcal{M}_{0}(R)$. Indeed, via [1], this follows from the localization theorem in $K$-theory. It suffices to show that $K_{0}\left(\operatorname{Mod}(R)_{>-\infty}^{\text {nil }}\right)=0$. This in turn follows because for any object $X \in \operatorname{Mod}(R)_{>-\infty}^{\text {nil }}, \bigoplus_{i=1}^{\infty} X \in \operatorname{Mod}(R)_{>-\infty}^{\text {nil }}$ as well, so that an Eilenberg swindle implies $K_{0}\left(\operatorname{Mod}(R)_{>-\infty}^{\text {nil }}\right)=0$. In particular, any truncated object in $\mathcal{M}(R)$ can be represented by an object of $\operatorname{Mod}(R)_{>-\infty}^{\mathrm{cpl}}$.

Remark 3.6. The constructions $\operatorname{Mod}(R)_{>-\infty}^{\mathrm{nil}}, \operatorname{Mod}(R)_{>-\infty}^{\mathrm{cpl}}$ are insensitive to replacing $R$ by its $I$-adic completion.

Construction 3.7 (The $I$-complete $t$-structure on $\mathcal{M}(R)$ ). The stable $\infty$-category $\operatorname{Mod}(R)_{>-\infty}^{\mathrm{cpl}}$ also admits a $t$-structure obtained by restriction from the usual $t$-structure on all $R$-modules, whose heart is given by derived $I$-complete discrete $\pi_{0}(R)$-modules; we refer to this as the $I$-complete $t$ structure. Similarly, using Construction 3.3. this t-structure descends to $\mathcal{M}_{0}(R)$. We observe that it also descends to $\mathcal{M}(R)$ with heart $\mathcal{B}(R)$. This follows because the $I$-torsion and $I$-complete $t$-structures on $\mathcal{M}_{0}(R)$ (and on $\operatorname{Mod}(R)_{>-\infty}^{\mathrm{cpl}}$ ) differ by a bounded amplitude. In particular, $\mathcal{M}(R)$ is also the left completion of $\mathcal{M}_{0}(R)$ with respect to the $I$-complete $t$-structure.

Construction 3.8 (Functoriality of $\mathcal{M}(R)$ ). The construction $R \mapsto \mathcal{M}(R)$ defines a functor in the connective $E_{\infty}$-ring $R$ (with choice of ideal $I \subset \pi_{0}(R)$ ) to the $\infty$-category of right-bounded, leftcomplete stable $\infty$-categories with $t$-structures (for either choice of $t$-structure), and right $t$-exact functors between them; on hearts it is the base-change functor on abelian categories $\mathcal{A}(R), \mathcal{B}(R)$. Finally, given any map $R \rightarrow R^{\prime}$ of connective $E_{\infty}$-rings, we get a right adjoint $\mathcal{M}\left(R^{\prime}\right) \rightarrow \mathcal{M}(R)$ to the base-change functor (given by restriction of scalars) which is $t$-exact.

3.2. Almost perfect complexes. Throughout this subsection, we use the $I$-complete $t$-structure and we assume $R$ is itself $I$-adically complete (no loss of generality by Remark [3.6). It will be necessary to compute some mapping spaces in $\mathcal{M}(R)$; for this, we use the following construction.

Construction 3.9 (Comparison of $\mathcal{M}(R)$ with the generic fiber). We have a $t$-exact functor (for the $I$-complete $t$-structure) $\operatorname{Mod}(R)_{>-\infty}^{\mathrm{cpl}} \rightarrow \mathrm{QCoh}(\operatorname{Spec}(R) \backslash V(I))$ given by restricting to the locus outside of $I$. This clearly annihilates $\operatorname{Mod}(R)_{>-\infty}^{\text {nil }}$, so we obtain a $t$-exact functor $\mathcal{M}_{0}(R) \rightarrow$ 
$\mathrm{QCoh}(\operatorname{Spec}(R) \backslash V(I))$. Since the target is left-complete, we obtain a factorization over a $t$-exact functor $j^{*}: \mathcal{M}(R) \rightarrow \mathrm{QCoh}(\operatorname{Spec}(R) \backslash V(I))$.

Proposition 3.10. Suppose $R$ is $I$-complete. Suppose $X, Y \in \operatorname{Mod}(R)_{>-\infty}^{\mathrm{cpl}}$. Suppose $X$ is $\leq I^{\infty}$ perfect to order $n+1$ and $Y$ is n-truncated in the $I$-complete $t$-structure on $\operatorname{Mod}(R)_{>-\infty}^{\mathrm{cpl}}$. Then the functor $j^{*}$ induces an equivalence of connective spectra $\tau_{\geq 0} \operatorname{Hom}_{\mathcal{M}_{0}(R)}(X, Y) \simeq \tau_{\geq 0} \operatorname{Hom}_{\mathrm{QCoh}(\operatorname{Spec}(R) \backslash V(I))}\left(j^{*} X, j^{*} Y\right)$.

Proof. The $R$-modules $\operatorname{Hom}_{\mathcal{M}_{0}(R)}(X, Y), \operatorname{Hom}_{\mathrm{QCoh}(\operatorname{Spec}(R) \backslash V(I))}\left(j^{*} X, j^{*} Y\right)$ are both local away from $I$ (i.e., belong to the image of the fully faithful embedding $\mathrm{QCoh}(\operatorname{Spec}(R) \backslash V(I)) \rightarrow \operatorname{Mod}(R))$, since the $\infty$-categories $\mathcal{M}_{0}(R), \mathrm{Q} \operatorname{Coh}(\operatorname{Spec}(R) \backslash V(I))$ are naturally tensored over $\operatorname{Perf}(\operatorname{Spec}(R) \backslash V(I))$. Thus, it suffices to show that $\tau_{\geq 0} \operatorname{Hom}_{\mathcal{M}_{0}(R)}(X, Y) \rightarrow \tau_{\geq 0} \operatorname{Hom}_{\mathrm{QCoh}(\operatorname{Spec}(R) \backslash V(I))}\left(j^{*} X, j^{*} Y\right)$ becomes an isomorphism after inverting any $t \in I$.

Note that the $R$-module $Y$ is $n$-truncated (with respect to the Postnikov $t$-structure). Therefore, the map $\tau_{\geq 0} \operatorname{Hom}_{\operatorname{Mod}(R)}(X, Y)[1 / t] \rightarrow \tau_{\geq 0} \operatorname{Hom}_{\operatorname{Mod}(R)}(X[1 / t], Y[1 / t])$ is an isomorphism by Proposition 2.23] since $X$ is $\leq I^{\infty}$-perfect to order $n+1$. To complete the proof, it suffices to show that for any $X^{\prime} \rightarrow X$ in $\operatorname{Mod}(R)_{>-\infty}^{\mathrm{cpl}}$ whose cofiber belongs to $\operatorname{Mod}(R)_{>-\infty}^{\mathrm{nil}}$, then the map

$$
\tau_{\geq 0} \operatorname{Hom}_{\operatorname{Mod}(R)_{>-\infty}^{\mathrm{cpl}}}\left(X^{\prime}, Y\right) \rightarrow \tau_{\geq 0} \operatorname{Hom}_{\operatorname{Mod}(R)_{>-\infty}^{\mathrm{cpl}}}(X, Y)
$$

induces an equivalence after inverting any $t \in I$. However, since $Y$ is $n$-truncated, we can identify this with the map $\tau_{\geq 0} \operatorname{Hom}_{\operatorname{Mod}(R)_{>-\infty}^{\mathrm{cpl}}}\left(\tau_{\leq n} X^{\prime}, Y\right) \rightarrow \tau_{\geq 0} \operatorname{Hom}_{\mathrm{Mod}(R)_{>-\infty}^{\mathrm{cpl}}}\left(\tau_{\leq n} X, Y\right)$ which becomes an isomorphism after inverting $t$, since $\tau_{\leq n} X^{\prime} \rightarrow \tau_{\leq n} X$ is a $\leq I^{\infty}$-isogeny.

Corollary 3.11. Suppose $R$ is I-complete. Suppose the ideal I is generated by g elements. Suppose $X \in \mathcal{M}(R)$ lives in degrees $\geq g$ with respect to the $I$-complete $t$-structure. Then $\pi_{0} \operatorname{Hom}_{\mathcal{M}(R)}(R, X)=$ 0 . Here we identify $R$ with the associated object of $\mathcal{M}(R)$.

Proof. When $X$ is truncated, this follows from Proposition 3.10 since $\operatorname{Spec}(R) \backslash V(I)$ has cohomological dimension $\leq g-1$ and truncated objects of $\mathcal{M}(R)$ and $\mathcal{M}_{0}(R)$ are identified. In general, we have that $\operatorname{Hom}_{\mathcal{M}(R)}(R, X)=\varliminf_{\lim _{n}} \operatorname{Hom}_{\mathcal{M}(R)}\left(R, \tau_{\leq n} X\right)$ from which the result follows via the Milnor exact sequence.

Our goal is to identify a subcategory of "weakly almost perfect objects" in $\mathcal{M}(R)$ and then to show that this subcategory is equivalent (via $\left.j^{*}\right)$ to the $\infty$-category $\operatorname{APerf}(\operatorname{Spec}(R) \backslash V(I))$.

Definition 3.12 (Objects weakly perfect to order $n$ ). We will say that an object of $\mathcal{M}_{0}(R)$ is weakly perfect to order $n$ if for any representative $X \in \operatorname{Mod}(R)_{>-\infty}^{\mathrm{cpl}}, X$ is $\leq I^{\infty}$-perfect to order $n$. This definition is clearly independent of the choice of representative $X$, since any two are related by a zig-zag of quasi-isogenies. We will say that an object is weakly almost perfect if it is weakly perfect to any order.

The condition that an object in $\mathcal{M}_{0}(R)$ should be weakly perfect to order $n$ depends only on its $n$-truncation with respect to the $I$-complete $t$-structure. Therefore, we also obtain analogous definitions of weakly almost perfect (resp. weakly perfect to order $n$ ) for objects of $\mathcal{M}(R)$.

Proposition 3.13. Suppose $R$ is I-complete. Let $X \in \mathcal{M}(R)_{\geq 0}$.

(1) $X$ is weakly perfect to order zero if and only if there exists a map $R^{r} \rightarrow X$ (for some $r \geq 0$ ) whose cofiber $C$ belongs to $\mathcal{M}(R)_{\geq 1}$. 
(2) Suppose $n>0$ and $X$ is weakly perfect to order $n-1$. Then $X$ is weakly perfect to order $n$ if and only if, for any (or every) map $R^{r} \rightarrow X$ with cofiber $C \in \mathcal{M}(R)_{\geq 1}$, we have that $C[-1]$ is weakly perfect to order $n-1$.

Proof. The condition that $X$ should be weakly perfect to order $n$ only depends on the $n$-truncation of $X$; moreover, maps $R^{r} \rightarrow X$ only depend on $\tau_{\leq g} X$ (Corollary 3.11), if $I$ is generated by $g$ elements. Therefore, without loss of generality we may assume that $X \in \mathcal{M}_{0}(R)_{<\infty}$ and can be represented as the image in $\mathcal{M}_{0}(R)$ of some $Y \in \operatorname{Mod}(R)_{>-\infty}^{\mathrm{cpl}}$, which without loss of generality we can take to be connective. Then $X$ is weakly perfect to order zero if and only if there exists a map $R^{r} \rightarrow Y$ whose cofiber in $\operatorname{Mod}(R)$ has $\pi_{0}$ which is $\leq I^{\infty}$-isogenous to zero, cf. Example 2.25. Assertion (1) now follows. Since any map of objects in $\mathcal{M}_{0}(R)$ can be represented by a map in $\operatorname{Mod}(R)_{>-\infty}^{\mathrm{cpl}}$ (subject to working up to quasiisogenies), assertion (2) follows by Proposition 2.26.

Proposition 3.14. (1) The collection of objects in $\mathcal{M}(R)_{\geq 0}$ which are weakly almost perfect is closed under finite colimits and geometric realizations.

(2) An object of $\mathcal{M}(R)_{\geq 0}$ is weakly almost perfect if and only if it can be written as a geometric realization of a simplicial object in $\mathcal{M}(R)_{\geq 0}$ each of whose terms is a finite direct sum of copies of $R$.

Proof. Part (1) follows from the fact that the condition of being $\leq I^{\infty}$-perfect to some order $n$ is closed under finite colimits and depends only on the $n$-truncation. Part (2) follows by iteration. Given a weakly almost perfect $X \in \mathcal{M}(R)_{\geq 0}$, one produces (using Proposition 3.13) a filtered object $0=P_{-1} \rightarrow P_{0} \rightarrow P_{1} \rightarrow \ldots$ such that $P_{i} / P_{i-1}$ is equivalent to a finite direct sum of copies of $R[i]$ and whose colimit (which exists for connectivity reasons) is $X$. Converting this into a simplicial object via the Dold-Kan correspondence [31, Sec. C.1.4], we see that (2) follows.

Proposition 3.15. Let $R$ be I-complete. Suppose $X, Y \in \mathcal{M}(R)$ and $X$ is weakly almost perfect. Then $j^{*} X \in \mathrm{QCoh}(\operatorname{Spec}(R) \backslash V(I))$ is almost perfect. Furthermore, the natural map induces an equivalence of $R$-module spectra

$$
\operatorname{Hom}_{\mathcal{M}(R)}(X, Y) \simeq \operatorname{Hom}_{\mathrm{Q} \operatorname{Coh}(\operatorname{Spec}(R) \backslash V(I))}\left(j^{*} X, j^{*} Y\right) .
$$

Proof. Any object in $\operatorname{Mod}(R)_{>-\infty}^{\mathrm{cpl}}$ which is $\leq I^{\infty}$-perfect to order $n$ restricts to an object of $\mathrm{QCoh}(\operatorname{Spec}(R) \backslash V(I))$ which is perfect to order $n$, so the first assertion follows. Since $\mathcal{M}(R)$ is left-complete, it suffices to show for each $n$ that

$$
\tau_{\geq 0} \operatorname{Hom}_{\mathcal{M}(R)}\left(\tau_{\leq n+1} X, \tau_{\leq n} Y\right) \stackrel{\sim}{\rightarrow} \tau_{\geq 0} \operatorname{Hom}_{\mathrm{QCoh}(\operatorname{Spec}(R) \backslash V(I))}\left(j^{*} \tau_{\leq n+1} X, j^{*} \tau_{\leq n} Y\right) ;
$$

these (connective) mapping spectra can be computed in $\mathcal{M}_{0}(R)_{\leq n}$. Thus, the result follows from Proposition 3.10 since we can represent $\tau_{\leq n+1} X$ by an $I$-complete $R$-module which is perfect to order $n+1$.

Proposition 3.16. The functor $j^{*}$ establishes a natural, symmetric monoidal equivalence between the weakly almost perfect objects of $\mathcal{M}(R)_{\geq 0}$ and $\operatorname{APerf}(\operatorname{Spec}(R) \backslash V(I))_{\geq 0}$. Similarly, $j^{*}$ establishes a natural, symmetric monoidal equivalence between the weakly almost perfect objects of $\mathcal{M}(R)$ and $\operatorname{APerf}(\operatorname{Spec}(R) \backslash V(I))$.

Proof. Full faithfulness is a consequence of Proposition 3.15. For essential surjectivity, it suffices to show that any object of $\operatorname{APerf}(\operatorname{Spec}(R) \backslash V(I))_{\geq 0}$ can be written as a geometric realization of a simplicial object which at each level is a direct sum of copies of the unit. This holds more generally for any quasi-affine spectral scheme (modeled on the spectrum of a connective $E_{\infty}$-ring). 
Indeed, let $\mathcal{F} \in \operatorname{APerf}(\operatorname{Spec}(R) \backslash V(I))_{\geq 0}$. Then by [31, Prop. 9.6.6.1], there is a map from a direct sum of copies of the unit to $\mathcal{F}$ inducing a surjection on $\pi_{0}$-sheaves. Since $\mathcal{F}$ is almost perfect and connective, we can assume that this is a finite direct sum. Continuing inductively as in the proof of Proposition 3.14, we can now write $\mathcal{F}$ as a geometric realization as desired. The assertions for $\mathcal{M}(R)$ follow from those for $\mathcal{M}(R)_{\geq 0}$.

\section{REVIEW OF MONADICITY AND DESCENT}

Our descent results take the form of an expression for a stable $\infty$-category as a homotopy limit of a cosimplicial stable $\infty$-category. In this section, we review some general results from [30, Sec. 4.7.5] for identifying such homotopy limits; these are closely related to the Barr-Beck-Lurie monadicity theorem.

Definition 4.1 (The Beck-Chevalley condition). Let $\mathcal{C} \bullet$ be an augmented cosimplicial $\infty$-category. We will say that $\mathcal{C}^{\bullet}$ satisfies the adjointability condition if for each $\alpha:[m] \rightarrow[n]$ in $\Delta^{+}$, the square

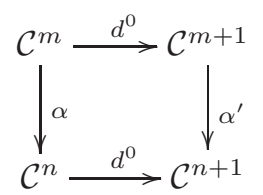

is right adjointable: that is, the horizontal arrows admit right adjoints (denoted $d_{*}^{0}$ ), and the induced Beck-Chevalley transformation $\alpha \circ d_{*}^{0} \rightarrow d_{*}^{0} \circ \alpha^{\prime}$ is an equivalence.

Example 4.2. Let $R \rightarrow R^{\prime}$ be a map of $E_{\infty}$-rings, and let $R^{\prime \bullet}$ be the associated Cech nerve (an augmented cosimplicial $E_{\infty}$-ring). Then the diagram of $\infty$-categories $\operatorname{Mod}\left(R^{\prime \bullet}\right)$ satisfies the adjointability condition. The reason is that if we have a pushout square of $E_{\infty}$-rings

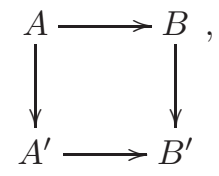

then the induced square after applying $\operatorname{Mod}(\cdot)$ is right adjointable.

For the next result, we let Cat ${ }_{\infty}$ denote the $\infty$-category of $\infty$-categories.

Theorem 4.3 (Lurie, 30, Corollary 4.7.5.3]). Let $\mathcal{C}^{\bullet}: \Delta^{+} \rightarrow$ Cat $_{\infty}$ be a functor. Suppose that:

(1) The (co)augmentation coaug: $\mathcal{C}^{-1} \rightarrow \mathcal{C}^{0}$ is conservative and has the following property: totalizations of coaug-split cosimplicial objects in $\mathcal{C}^{-1}$ exist and are preserved by coaug.

(2) $\mathcal{C} \bullet$ satisfies the adjointability condition.

Then $\mathcal{C}^{\bullet}$ is a limit diagram.

In practice, the adjointability condition will be automatic (from e.g., Example 4.2), so to verify that certain diagrams are limit diagrams, it will be necessary to verify condition (1) of Theorem 4.3 . There will be two basic tools: universal descent maps (for which condition (1) will hold for essentially diagrammatic reasons) and situations where one has a $t$-structure.

We begin with the universal descent case. The following definition is essentially from [32, Sec. 3] and [31, Sec. D.3]. The main example is given by base-change along a universal descent morphism of $E_{\infty}$-rings. 
Definition 4.4 (Universal descent functors). Let $\mathcal{C}, \mathcal{D}$ be idempotent-complete, stable $\infty$-categories. We say that an exact functor $f: \mathcal{C} \rightarrow \mathcal{D}$ is of universal descent if there exists an exact functor $\varphi: \mathcal{C} \rightarrow \mathcal{C}$ with the following properties:

(1) The identity functor id $\mathrm{i}_{\mathcal{C}}$ is a retract of $\varphi$.

(2) There exists a filtration in the $\infty$-category of functors $\operatorname{Fun}(\mathcal{C}, \mathcal{C})$,

$$
\varphi_{0} \rightarrow \varphi_{1} \rightarrow \cdots \rightarrow \varphi_{e}=\varphi
$$

such that each $\varphi_{i} / \varphi_{i-1} \in \operatorname{Fun}(\mathcal{C}, \mathcal{C})$ can be written as $\psi_{i} \circ f$ for some $\psi_{i} \in \operatorname{Fun}(\mathcal{D}, \mathcal{C})$ exact.

In the following, we denote by $\mathrm{Cat}_{\infty}^{\text {perf }}$ the $\infty$-category of idempotent-complete, stable $\infty$ categories and exact functors between them.

Proposition 4.5. Let $\mathcal{C}^{\bullet}: \Delta^{+} \rightarrow \mathrm{Cat}_{\infty}^{\text {perf }}$ be a functor satisfying the adjointability condition. Suppose coaug: $\mathcal{C}^{-1} \rightarrow \mathcal{C}^{0}$ is universal descent. Then $\mathcal{C}^{\bullet}$ is a limit diagram.

Proof. It remains to verify condition (1) of Theorem 4.3. First, the filtration (6) implies that any object in the kernel of coaug vanishes, so coaug is conservative. Next, consider the collection $\mathfrak{V}$ of cosimplicial objects $Y^{\bullet} \in \operatorname{Fun}\left(\Delta, \mathcal{C}^{-1}\right)$ which admit a totalization in $\mathcal{C}^{-1}$ and which is preserved under coaug. Clearly $\mathfrak{V}$ is a thick subcategory of $\operatorname{Fun}\left(\Delta, \mathcal{C}^{-1}\right)$, and it contains those cosimplicial objects which admit splittings. If $X^{\bullet} \in \operatorname{Fun}\left(\Delta, \mathcal{C}^{-1}\right)$ is such that $\operatorname{coaug}\left(X^{\bullet}\right)$ admits a splitting, then our assumption implies that $X^{\bullet}$ belongs to $\mathfrak{V}$, whence the claim.

Next, we prove a descent criterion in the case of $t$-structures. This is essentially a version of the argument used for faithfully flat descent in [31, Sec. D.6].

Proposition 4.6 (Descent criterion for left-complete stable $\infty$-categories). Let $\mathcal{C} \bullet$ be an augmented cosimplicial object of $\mathrm{Cat}_{\infty}^{\text {perf }}$. Suppose that each $\mathcal{C}^{i}, i \geq-1$ is equipped with a right-bounded, leftcomplete $t$-structure, and the following conditions are satisfied.

(1) $\mathcal{C}^{\bullet}$ satisfies the adjointability condition.

(2) Each cosimplicial structure map is right t-exact, and has a t-exact right adjoint.

(3) The coaugmentation functor coaug: $\mathcal{C}^{-1} \rightarrow \mathcal{C}^{0}$ is conservative and t-exact.

Then $\mathcal{C}^{\bullet}$ is a limit diagram. Similarly for $\mathcal{C}_{\geq 0}^{\bullet}$ and $\mathcal{C}_{[m, n]}^{\bullet}$ for any $m \leq n$.

Proof. For each $m \leq n$, the hypotheses of the proposition yield (from $\mathcal{C}^{\bullet}$ ) an augmented cosimplicial $\infty$-category $\mathcal{C}_{[m, n]}^{\bullet}$; the cosimplicial structure maps are obtained by truncating those of $\mathcal{C}^{\bullet}$. Moreover, the hypotheses show that $\mathcal{C}_{[m, n]}^{\bullet}$ satisfies the adjointability condition, and that $\mathcal{C}_{[m, n]}^{-1} \rightarrow \mathcal{C}_{[m, n]}^{0}$ preserves totalizations and is conservative. Therefore, by Theorem 4.3 we find that $\mathcal{C}_{[m, n]}^{\bullet}$ is a limit diagram. Taking the limit over $n$ and the colimit over $m$, we obtain that $\mathcal{C}^{\bullet}$ is a limit diagram. A similar argument works for $\mathcal{C}_{\geq 0}^{\bullet}$.

Example 4.7 (Derived faithfully flat descent). Let $A \rightarrow B$ be a faithfully flat map of commutative rings (or more generally of connective $E_{\infty}$-rings). Then, via the Postnikov $t$-structures, we can apply Proposition 4.6 to obtain an equivalence (where $>-\infty$ denotes bounded-below objects)

$$
\operatorname{Mod}(A)_{>-\infty} \simeq \lim _{\longleftarrow}\left(\operatorname{Mod}(B)_{>-\infty} \rightrightarrows \operatorname{Mod}\left(B \otimes_{A} B\right)_{>-\infty} \underset{\rightarrow}{\rightarrow} \cdots\right) .
$$

This is the derived version of faithfully flat descent from [31, Cor. D.6.3.3] (at least in the boundedbelow case), and the above argument is that of loc. cit. 


\section{The Universal Descent CASE}

In this section, we show (Theorem 5.8 ) that almost perfect complexes on $\operatorname{Spec}\left(\widehat{R}_{I}\right) \backslash V(I R)$ form a sheaf with respect to the universal descent topology (Definition 5.1). As an application, we prove arc-descent results for the category of finite étale covers, extending results of [8].

\subsection{The descent theorem.}

Definition 5.1 (Universal descent morphisms). Let $f: R \rightarrow R^{\prime}$ be a morphism of $E_{\infty}$-rings. We will say that $f$ is a universal descent morphism if the base-change functor $\operatorname{Mod}(R) \rightarrow \operatorname{Mod}\left(R^{\prime}\right)$ is a universal descent functor in the sense of Definition 4.4. Alternatively, this holds if and only if the thick subcategory of $\operatorname{Mod}(R)$ generated by the $R^{\prime}$-modules is all of $\operatorname{Mod}(R)$. Using this notion, we obtain the universal descent topology on the opposite of the $\infty$-category of $E_{\infty}$-rings.

Remark 5.2. The class of universal descent morphisms is closed under composition. Moreover, if a composite $R \rightarrow S \rightarrow T$ of morphisms of $E_{\infty}$-rings is a universal descent morphism, then $R \rightarrow S$ is a universal descent morphism.

Definition 5.3 (Exponents of universal descent morphisms). Suppose $f: R \rightarrow R^{\prime}$ is a universal descent morphism. Let $R^{\prime \bullet}$ be the Cech nerve. Then $R \simeq \operatorname{Tot}\left(R^{\prime \bullet}\right)$. Moreover, the associated Tot-tower $\left\{\operatorname{Tot}^{n}\left(R^{\prime \bullet}\right)\right\}$ defines a constant pro-object of $\operatorname{Mod}(R)$, so there exists $e \geq 0$ such that $R$ is a retract of the partial totalization $\operatorname{Tot}^{e}\left(R^{\prime \bullet}\right)$; the smallest such $e$ is called the exponent.

This class of morphisms was studied in [32] and [31, Sec. D.3], to which we refer for more details; see also [33] for a survey. In particular, one has the following basic result (a special case of Proposition 4.5):

Theorem 5.4. Given a universal descent morphism $R \rightarrow R^{\prime}$, the natural map $\operatorname{Mod}(R) \rightarrow \underset{\lim }{\longleftarrow}\left(\operatorname{Mod}\left(R^{\prime}\right) \rightrightarrows\right.$ $\left.\operatorname{Mod}\left(R^{\prime} \otimes_{R} R^{\prime}\right) \underset{\rightarrow}{\rightarrow} \ldots\right)$ is an equivalence. In other words, $\operatorname{Mod}(\cdot)$ is a sheaf for the universal descent topology.

In the case of finitely presented morphisms of discrete rings, one can give a concrete geometric criterion for a map to be universal descent, due to Bhatt-Scholze [10, Prop. 11.25] in the noetherian case; here we observe that it holds generally.

Theorem 5.5. Let $f: R \rightarrow R^{\prime}$ be a finitely presented map of discrete rings. Suppose $f$ is a $v$-cover ([37] and [10, Sec. 2]). Then $f$ is a universal descent morphism.

Proof. By [37. Theorem 6.4], it follows that $f$ is obtained as the (underived) base-change of a $v$-cover of finitely presented $\mathbb{Z}$-algebras, $R_{0} \rightarrow R_{0}^{\prime}$ along a map $R_{0} \rightarrow R$. In particular, $R^{\prime}=\tau_{\leq 0}\left(R \otimes_{R_{0}} R_{0}^{\prime}\right)$. The map $R_{0} \rightarrow R_{0}^{\prime}$ is a universal descent morphism [10, Prop. 11.25]. Now the map (of $E_{\infty}-$ rings) $R \rightarrow R \otimes_{R_{0}} R_{0}^{\prime}$ is universal descent by base-change. By Lemma 5.6, it follows that $R \rightarrow R^{\prime}$ is a universal descent morphism.

Lemma 5.6. Let $f: R \rightarrow S$ be a map of connective $E_{\infty}$-rings which is universal descent. Suppose $R$ is discrete. Then the composite map $R \rightarrow S \rightarrow \pi_{0}(S)$ is universal descent.

Proof. We use throughout the following basic observation, valid since $R$ is discrete. Let $R \rightarrow M$ be a map of $R$-modules; then this map admits a section if and only if the composite $R \rightarrow M \rightarrow \tau_{\leq 0} M$ admits a section.

Let $S^{\bullet}$ be Čech nerve of $R \rightarrow S$. Then for some $N, R$ is a retract of $\operatorname{Tot}^{N}\left(S^{\bullet}\right)$; equivalently, the map $R \rightarrow \operatorname{Tot}^{N}\left(S^{\bullet}\right) \rightarrow \tau_{\leq 0}\left(\operatorname{Tot}^{N}\left(S^{\bullet}\right)\right)$ admits a section. Now the map $\tau_{\leq 0}\left(\operatorname{Tot}^{N}\left(S^{\bullet}\right)\right) \rightarrow$ 
$\tau_{\leq 0}\left(\operatorname{Tot}^{N}\left(\tau_{\leq N+1}\left(S^{\bullet}\right)\right)\right)$ is an equivalence. Therefore, the map $R \rightarrow \tau_{\leq 0}\left(\operatorname{Tot}^{N}\left(\tau_{\leq N+1}\left(S^{\bullet}\right)\right)\right)$ admits a section, and hence the map $R \rightarrow \operatorname{Tot}^{N}\left(\tau_{\leq N+1}\left(S^{\bullet}\right)\right)$ admits a section too. It follows that $R$ is a retract of a finite limit of a diagram of $R$-modules, each of which admits the structure of a $\tau_{\leq N+1} S$ module. Consequently $R \rightarrow \tau_{\leq N+1} S$ is a universal descent morphism. Via the Postnikov tower, we see that $\tau_{\leq N+1} S \rightarrow \pi_{0}(S)$ is a universal descent morphism. Composing, the claim follows.

Proposition 5.7. Let $R \rightarrow R^{\prime}$ be a universal descent map of $E_{\infty}$-rings, and let $I \subset \pi_{0}(R)$ be a finitely generated ideal. Then $\hat{R} \rightarrow \hat{R}^{\prime}$ is universal descent.

Proof. This follows because we can write $R$ as a retract of a finite limit of a diagram of $R$-modules admitting the structure of $R^{\prime}$-module, and then complete everywhere.

The main descent theorem that we prove in this section is the following.

Theorem 5.8 (Rigid analytic descent in the universal case). Let $R$ be a connective $E_{\infty}$-ring and let $I \subset \pi_{0}(R)$ be a finitely generated ideal. Then, on the $\infty$-category of connective $E_{\infty}-R$-algebras, the construction $R^{\prime} \mapsto \operatorname{APerf}\left(\operatorname{Spec}\left(\widehat{R}_{I}^{\prime}\right) \backslash V(I)\right)$ is a sheaf for the universal descent topology. Similarly for $R^{\prime} \mapsto \operatorname{APerf}\left(\operatorname{Spec}\left({\widehat{R^{\prime}}}_{I}\right) \backslash V(I)\right)_{\geq 0}$ and with Perf replacing APerf.

The proof of Theorem 5.8 is based on showing that $R^{\prime} \mapsto \mathcal{M}\left(R^{\prime}\right)$ is a sheaf for the universal descent topology, and that the property of an object in $\mathcal{M}\left(R^{\prime}\right)$ being weakly almost perfect is local.

Proposition 5.9. The construction $R^{\prime} \mapsto \mathcal{M}\left(R^{\prime}\right)$ is a sheaf for the universal descent topology.

Proof. Given a universal descent map $S \rightarrow S^{\prime}$ of connective $E_{\infty}$ - $R$-algebras, we form the Čech nerve $S^{\bullet}$, an augmented cosimplicial ring. The augmented cosimplicial stable $\infty$-category $\operatorname{Mod}\left(S^{\bullet}\right)_{>-\infty}^{\mathrm{cpl}}$ satisfies the adjointability condition; therefore, so does $\mathcal{M}\left(S^{\bullet}\right)$ since the relevant adjoints pass through the procedure that constructs $\mathcal{M}(S)$ from $\operatorname{Mod}(S)_{>-\infty}^{\mathrm{cpl}}$. Now $\mathcal{M}(S) \rightarrow \mathcal{M}\left(S^{\prime}\right)$ is a universal descent functor, by our assumption that $S \rightarrow S^{\prime}$ is universal descent as a map of $E_{\infty}$-rings. Therefore, the result follows from Proposition 4.5.

Proposition 5.10. Let $R \rightarrow R^{\prime}$ be a universal descent map of connective $E_{\infty}$-rings of exponent e. Let $M \in \operatorname{Mod}(R)$ be bounded-below. Suppose $R^{\prime} \otimes_{R} M$ is $\leq I^{r}$-perfect to order $n$. Then $M$ is $\leq I^{\text {re }}$-perfect to order $n-e-1$.

Proof. For any filtered category $\mathcal{I}$ and functor $\mathcal{I} \rightarrow \operatorname{Mod}(R)_{\leq n-e-1}, j \mapsto N_{j}$, we will verify that the natural map

$$
\omega: \underset{J}{\lim } \tau_{\geq 0} \operatorname{Hom}_{R}\left(M, N_{j}\right) \rightarrow \tau_{\geq 0} \operatorname{Hom}_{R}\left(M, \underset{J}{\lim } N_{j}\right)
$$

is an $\leq I^{r e}$-isogeny (compare Proposition 2.24). More generally, for each functor $f: \mathcal{I} \rightarrow \operatorname{Mod}(R)$, we consider the map $\omega=\omega(f)$ of (77). Our hypothesis implies that if $f$ lifts to a functor with values in $\operatorname{Mod}\left(R^{\prime}\right)_{\leq n-1}$, then $\omega(f)$ is a $\leq I^{r}$-isogeny.

Let $R^{\prime \bullet}$ be the Čech nerve of $R \rightarrow R^{\prime}$, considered as a cosimplicial $R$-module. For any $R$-module $N$, we can write $N$ as a functorial retract of $\operatorname{Tot}^{e}\left(N \otimes_{R} R^{\bullet \bullet}\right)$. It follows that if $N \in \operatorname{Mod}(R)_{\leq n-e-1}$, then we can write $N$ as a functorial retract of $\operatorname{Tot}^{e}\left(\tau_{\leq n-1}\left(N \otimes_{R} R^{\prime \bullet}\right)\right)$.

Now returning to (77) in the case where $f$ takes values in $\leq n-e-1$-truncated modules, we find that $\omega(f)$ is a retract of a partial totalization $\operatorname{Tot}^{e}$ of $\omega\left(\tau_{\leq n-1}\left(f \otimes_{R} R^{\prime \bullet}\right)\right)$. From the above, it follows that $\omega(f)$ is a retract of a partial totalization $\operatorname{Tot}^{e}$ of a diagram of $\leq I^{r}$-isogenies, whence the claim. 
Proposition 5.11. Let $R \rightarrow R^{\prime}$ be a universal descent map of $I$-complete connective $E_{\infty}$-rings of exponent e. Let $M \in \operatorname{Mod}(R)$ be I-complete and bounded-below. Suppose $\widehat{R^{\prime} \otimes_{R}} M \in \operatorname{Mod}\left(R^{\prime}\right)$ is $\leq I^{\infty}$-perfect to order $n$. Then $M$ is $\leq I^{\infty}$-perfect to order $n-e-1$.

Proof. Fix a tower $\left\{R_{m}\right\}$ as in Construction 2.32, By Proposition 2.35, it suffices to show that there exists $r$ such that $M \otimes_{R} R_{m}$ is $\leq I^{r}$-perfect to order $n-e-1$ for all $m$. But by assumption, there exists $r^{\prime}$ such that $\left(M \otimes_{R} R_{m}\right) \otimes_{R} R^{\prime}$ is $\leq I^{r^{\prime}}$-perfect to order $n$ for all $m$. Now apply Proposition 5.10 (with $r=r^{\prime} e$ ) to conclude.

Corollary 5.12. Let $R \rightarrow R^{\prime}$ be a universal descent map of I-complete connective $E_{\infty}$-rings of exponent e. Let $M \in \mathcal{M}(R)$. If $M \otimes_{R} R^{\prime}$ is weakly perfect to order $n$, then $M$ is weakly perfect to order $n-e-1$.

Proof. This follows from Proposition 5.11 since we can (up to truncating homotopy groups in high enough degrees) assume that $M$ is represented by an object of $\operatorname{Mod}(R)_{>-\infty}^{\mathrm{cpl}}$.

Proof of Theorem 5.8. We first prove the results for Perf, APerf. Since Perf is the subcategory of dualizable objects in APerf, it suffices to prove the result for APerf. We have seen that the construction $R^{\prime} \mapsto \mathcal{M}\left(R^{\prime}\right)$ is a sheaf for the universal descent topology (Proposition [5.9), and there is a natural fully faithful embedding $\operatorname{APerf}\left(\operatorname{Spec}\left(\hat{R}^{\prime}\right) \backslash V(I)\right) \subset \mathcal{M}\left(R^{\prime}\right)$ (Proposition 3.16) with image the weakly almost perfect objects. It suffices now to show that the property of belonging to the image of this embedding (i.e., being weakly almost perfect) is local in the universal descent topology. But this follows from Corollary 5.12. Note that it suffices to work everywhere with $I$-complete $E_{\infty}$-rings (Proposition 5.7).

To obtain the results for $\operatorname{APerf}_{\geq 0}$ (and hence Perf $\geq 0$ ), it suffices to show that for a universal descent map $R^{\prime} \rightarrow S^{\prime}$ of $E_{\infty}-R$-algebras and an object $M \in \operatorname{APerf}\left(\operatorname{Spec}\left(\hat{R}^{\prime} \backslash V(I)\right)\right)$ whose pullback to $\operatorname{APerf}\left(\operatorname{Spec}\left(\hat{S}^{\prime} \backslash V(I)\right)\right.$ is connective, then $M$ is connective. This is a local question, and $\hat{R}^{\prime} \rightarrow \hat{S}^{\prime}$ is of universal descent. Thus, it suffices to verify the following: if $A \rightarrow B$ is a universal descent map of connective $E_{\infty}$-algebras and $M \in \operatorname{Mod}(A)$ is almost perfect, then $M$ is connective if and only if $M \otimes_{A} B$ is connective. By induction, we can assume $M$ is $(-1)$-connective. But then $\pi_{-1}(M)$ is a finitely presented $\pi_{0}(A)$-module and $\pi_{-1}(M) \otimes_{\pi_{0}(A)} \pi_{0}(B)=0$. Since $\pi_{0}(A) \rightarrow \pi_{0}(B)$ is surjective on spectra, this forces (by Nakayama) $\pi_{-1}(M)=0$ as desired.

5.2. arc-descent. Let $R$ be a $\mathbb{Z}\left[x_{1}, \ldots, x_{n}\right]$-algebra. Our goal in this section is to study the descent properties of the functor

$$
R \mapsto \mathcal{F}(R) \stackrel{\text { def }}{=}\left\{\text { finite étale schemes over } \operatorname{Spec}\left(\hat{R}_{\left(x_{1}, \ldots, x_{n}\right)} \backslash V\left(x_{1}, \ldots, x_{n}\right)\right)\right\},
$$

as a functor from $\mathbb{Z}\left[x_{1}, \ldots, x_{n}\right]$-algebras to categories.

Remark 5.13 (Derived completions versus completions). In (8), as usual, the notation $\widehat{R}_{\left(x_{1}, \ldots, x_{n}\right)}$ refers to the derived completion (which is a connective $E_{\infty}$-ring). One can replace the derived completion with the classical completion. For any $R$, there is a map from the derived completion $\widehat{R}_{\left(x_{1}, \ldots, x_{n}\right)}$ to the classical completion $\hat{R}_{\left(x_{1}, \ldots, x_{n}\right)}^{\mathrm{cl}}$. The induced map on $\pi_{0}$ is surjective, and the kernel squares to zero; this follows from the lim-spectral sequence (i.e., the Milnor exact sequence in this case). Alternatively, this fact follows directly from Lemma 2.28, Consequently, finite étale schemes are the same whether one uses the classical or derived completion. Similarly, in this section, there is no extra generality gained by working with connective $E_{\infty}$-rings rather than discrete rings. 
We use the following fundamental algebrization result, which allows one to replace the completion with the henselization. For a qcqs scheme $X$, we let $\operatorname{FEt}(X)$ be the category of finite étale schemes over $X$.

Theorem 5.14 (Elkik [20, Gabber-Ramero [23, Prop. 5.4.54], Gabber [27, Th. 2.1.2]). For any $\mathbb{Z}\left[x_{1}, \ldots, x_{n}\right]$-algebra $R$ which is henselian along $\left(x_{1}, \ldots, x_{n}\right)$, the natural functor

$$
\operatorname{FEt}\left(\operatorname{Spec}(R) \backslash V\left(x_{1}, \ldots, x_{n}\right)\right) \rightarrow \operatorname{FEt}\left(\operatorname{Spec}\left(\hat{R}_{x_{1}, \ldots, x_{n}}\right) \backslash V\left(x_{1}, \ldots, x_{n}\right)\right)
$$

is an equivalence of categories.

Corollary 5.15. The functor $\mathcal{F}$ is finitary, i.e., commutes with filtered colimits.

Proof. This follows from Theorem [5.14, since henselization commutes with filtered colimits.

We now use the following definition, from 8$]$ and due independently to Rydh. It is a refinement of the $v$-topology [37, 10, which in turn is the non-noetherian version of Voevodsky's $h$-topology. We also note a slight variant of it, as in [8, Sec. 6.2].

Definition 5.16 (arc-covers). A map of commutative rings $R \rightarrow R^{\prime}$ is said to be an arc-cover if for every rank $\leq 1$-valuation ring $V$ and map $R \rightarrow V$, there is an extension of rank $\leq 1$-valuation rings $V \rightarrow V^{\prime}$ and a commutative diagram

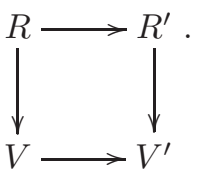

This defines the arc-topology on the category of affine schemes.

Definition 5.17 (The $\operatorname{arc}_{\left(x_{1}, \ldots, x_{n}\right)}$-topology). A map $R \rightarrow R^{\prime}$ of $\mathbb{Z}\left[x_{1}, \ldots, x_{n}\right]$-algebras is said to be an $\operatorname{arc}_{\left(x_{1}, \ldots, x_{n}\right)}$-cover if for every rank $\leq 1$ valuation ring $V$ with map $R \rightarrow V$ such that the image of $\left(x_{1}, \ldots, x_{n}\right)$ in $V$ is nonzero but contained in the maximal ideal, there exists an extension of rank $\leq 1$-valuation rings $V \rightarrow V^{\prime}$ and a commutative diagram as in (9). This defines the $\operatorname{arc}_{\left(x_{1}, \ldots, x_{n}\right)}$-topology on the category of affine schemes over Spec $\mathbb{Z}\left[x_{1}, \ldots, x_{n}\right]$.

Remark 5.18. A map $R \rightarrow R^{\prime}$ is an $\operatorname{arc}_{\left(x_{1}, \ldots, x_{n}\right)}$-cover if and only if $R \rightarrow R^{\prime} \times R /\left(x_{1}, \ldots, x_{n}\right) \times$ $R\left[1 / x_{1}\right] \times \cdots \times R\left[1 / x_{n}\right]$ is an arc-cover. Therefore, a functor $\mathcal{G}$ is a sheaf for the $\operatorname{arc}_{\left(x_{1}, \ldots, x_{n}\right)}$-topology if and only if it is a sheaf for the arc-topology and $\mathcal{G}\left(R /\left(x_{1}, \ldots, x_{n}\right)\right), \mathcal{G}\left(R\left[1 / x_{i}\right]\right)$ are the terminal object for any $\mathbb{Z}\left[x_{1}, \ldots, x_{n}\right]$-algebra $R$.

We refer to [8, Ex. 6.15] for a discussion of the relationship between the $\operatorname{arc}_{x}$-topology (so $n=1$ in the above) and surjectivity at the level of adic spectra.

Theorem 5.19. The functor $R \mapsto \mathcal{F}(R)$ is a sheaf for the $\operatorname{arc}_{\left(x_{1}, \ldots, x_{n}\right)}$-topology.

Informally, Theorem 5.19 is an expression of a (well-known) principle that the theory of (purely algebraically defined) finite étale schemes behaves well in analytic geometry, and satisfies very strong descent results; many cases of this result are already in the literature. For instance, for perfectoid spaces, $v$-descent of finite étale schemes appears as [39, Prop. 9.7]. In the case of abelian étale covers, this result appears (and more generally for the higher cohomology) in [8. Cor. 6.17] (with the restriction to a principal ideal, but this is not necessary). Theorem 5.19 as stated can also be proved using Gabber's rigidity results for nonabelian cohomology, cf. [22] and [27, Exp. XX].

We explain here a quick proof of Theorem 5.19 using the theory of finite étale algebra objects in a symmetric monoidal stable $\infty$-category ([36, 32]) and Theorem 5.8 . 
Construction 5.20 (Finite étale algebra objects). For a small, idempotent-complete stably symmetric monoidal $\infty$-category $\mathcal{C}$, one extracts [32, Def. 6.1] a category $\operatorname{FEt}(\mathcal{C})$ of "finite étale algebra objects" of $\mathcal{C}$, a full subcategory of the $\infty$-category $\operatorname{CAlg}(\mathcal{C})$ of commutative algebra objects of $\mathcal{C} 2$ In the case where $\mathcal{C}=\operatorname{Perf}(X)$ for $X$ a qcqs spectral scheme (modeled on the spectra of connective $E_{\infty}$-rings), then $\mathrm{FEt}(\mathcal{C})$ recovers precisely the opposite to the category of finite étale schemes over $X$ (or equivalently of the underlying scheme $\pi_{0} X$ ).

Example 5.21 (Torsors in $\operatorname{FEt}(\mathcal{C})$ ). Let $G$ be a finite group. A $G$-torsor in $\operatorname{FEt}(\mathcal{C})$ is given by an object $A \in \operatorname{Fun}(B G, \operatorname{CAlg}(\mathcal{C})$ (i.e., a commutative algebra equipped with a $G$-action) such that:

(1) As an object of $\operatorname{Fun}(B G, \mathcal{C}), A$ belongs to the thick subcategory generated by the induced $G$ objects (i.e., the $G$-action is nilpotent; compare the discussion in 33, Sec. 4]). In particular, $A^{h G}$ exists in $\mathcal{C}$.

(2) The natural map $\mathbf{1} \rightarrow A^{h G}$ is an equivalence.

(3) The shearing map $A \otimes A \rightarrow \prod_{G} A$ is an equivalence in $\mathcal{C}$.

These conditions are due to Rognes [36, who introduces the notion of a Galois extension of an $E_{\infty}$-ring.

Remark $5.22(\mathrm{FEt}(\cdot)$ preserves some limits). In general, the construction $\mathcal{C} \mapsto \operatorname{FEt}(\mathcal{C})$ need not preserve limits: the construction $\mathcal{C} \mapsto \operatorname{CAlg}(\mathcal{C})$ does, but condition (1) involved may not. However, suppose we have an augmented cosimplicial stably symmetric monoidal $\infty$-category $\mathcal{C}^{\bullet}$ which is a limit diagram; suppose moreover that $\mathcal{C}^{-1} \rightarrow \mathcal{C}^{0}$ is a universal descent morphism. Then $\operatorname{FEt}\left(\mathcal{C}^{-1}\right) \simeq \lim \left(\operatorname{FEt}\left(\mathcal{C}^{\bullet}\right)\right)$. This follows by considering $G$-torsors for each finite group $G$ and 32 , Cor. 5.40].

Proof of Theorem 5.19. By Remark 5.18, it is sufficient to verify that $\mathcal{F}$ is an arc-sheaf. We will apply the criterion of $[\underline{8}$, Theorem 4.1] to the functor $\mathcal{F}$. First, note that the functor $\mathcal{F}$ is finitary thanks to Corollary 5.15 .

We show that $\mathcal{F}$ satisfies $h$-descent. Let $R \rightarrow R^{\prime}$ be an $h$-cover, i.e., a finitely presented $v$-cover. It suffices to show that in this case,

$$
\mathcal{F}(R) \simeq \lim _{\longleftarrow}\left(\mathcal{F}\left(R^{\prime}\right) \rightrightarrows \mathcal{F}\left(\pi_{0}\left(R^{\prime} \otimes_{R} R^{\prime}\right)\right) \underset{\rightarrow}{\rightarrow} \cdots\right)
$$

Indeed, by Theorem 5.5, $R \rightarrow R^{\prime}$ is a universal descent morphism, so by Theorem 5.8 we have a limit diagram

$$
\operatorname{Perf}\left(\operatorname{Spec}\left(\widehat{R}_{I}\right) \backslash V(I)\right) \simeq \lim _{\longleftarrow}\left(\operatorname{Perf}\left(\operatorname{Spec}\left({\widehat{R^{\prime}}}_{I}\right) \backslash V(I)\right) \rightrightarrows \operatorname{Perf}\left(\operatorname{Spec}\left(\widehat{R^{\prime} \otimes_{R} R_{I}^{\prime}}\right) \backslash V(I)\right) \ldots\right)
$$

(involving the iterated derived tensor products of $R^{\prime}$ over $R$ ). Applying Construction [5.20, and noting that the higher homotopy groups in the derived tensor product do not affect finite étale schemes, (10) follows (also via Remark 5.22).

To check the criterion of 8 . Theorem 4.1], it suffices to check the "aic-v-excision" condition of loc. cit. Suppose $V$ is an absolutely integrally closed valuation ring and $\mathfrak{p} \subset V$ a prime ideal; we

\footnotetext{
${ }^{2}$ The definition in loc. cit. is stated for a presentably symmetric monoidal $\infty$-category; however, we can embed $\mathcal{C}$ into $\operatorname{Ind}(\mathcal{C})$.
} 
need to show that the square

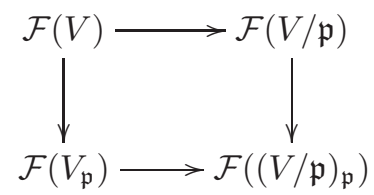

is a pullback. To see this, we consider the map $V \rightarrow V / \mathfrak{p} \times V_{\mathfrak{p}}$, which is of universal descent because of the pullback description $V \simeq V / \mathfrak{p} \times_{(V / \mathfrak{p})_{\mathfrak{p}}} V_{\mathfrak{p}}$. It follows as in the previous paragraph that $\mathcal{F}$ carries the Čech nerve of this map to a limit diagram; unwinding the Čech nerve now shows that (11) is a pullback square as desired.

\section{EXAMPLE: FLAT DESCENT ON CLASSICAL RIGID SPACES}

Throughout this section, we fix a complete nonarchimedean field $K$ with ring of integers $\mathcal{O}_{K} \subset K$, and a nonzero nonunit $\pi \in \mathcal{O}_{K}$. We will work in the setting of classical rigid geometry and $K$-affinoid algebras ${ }^{3}$ In this section, we give a proof of Theorem 1.4 from the introduction (as Theorem 6.16 below), i.e., of flat descent of almost perfect complexes for $K$-affinoid algebras. This result can also be deduced from the case of coherent sheaves [13, although our argument will be independent; cf. also 25 for the case of étale descent.

We freely use the flattening results of Bosch-Lütkebohmert 14 and various finiteness properties of topologically finitely presented $\mathcal{O}_{K}$-algebras, but otherwise the methods make no reference to results of rigid geometry (e.g., Kiehl's theorem that coherent modules satisfy descent in the analytic topology; these methods recover Kiehl's theorem). Our main observation (Proposition 6.14) is that if $A \rightarrow B$ is a faithfully flat map of affinoid $K$-algebras, then the map $A_{0} \rightarrow B_{0}$ of appropriate rings of definition is a universal descent morphism; furthermore, the iterated derived tensor products of $B_{0}$ over $A_{0}$ have higher homotopy which is bounded $\pi$-power torsion. The result will then follow from Theorem 5.8

6.1. Review of coherent rings. In the following it will be necessary to work with coherent (and especially stably coherent) rings; these are rings for which many of the convenient module-theoretic finiteness properties of noetherian rings still hold, provided one restricts to finitely presented modules. See 24 for a textbook reference.

Definition 6.1 (Coherent rings). A ring $R$ is called coherent if the category of finitely presented $R$-modules is abelian (equivalently, stable under kernels). This holds if and only if each finitely generated ideal $I \subset R$ is finitely presented as an $R$-module. A commutative ring $R$ is called stably coherent if every finitely presented $R$-algebra is coherent.

Definition 6.2 (Coherent $E_{\infty}$-rings). Let $R$ be a connective $E_{\infty}$-ring. We say that $R$ is coherent (resp. stably coherent) if $\pi_{0}(R)$ is coherent (resp. stably coherent) as a commutative ring and each $\pi_{i}(R), i \geq 0$ is finitely presented as a $\pi_{0}(R)$-module.

Remark 6.3 (Characterization of almost perfect complexes). Suppose $R$ is a coherent $E_{\infty}$-ring. In this case, a bounded-below $R$-module spectrum $M \in \operatorname{Mod}(R)$ is almost perfect if and only if the homotopy groups $\pi_{i}(M)$ are finitely presented $R$-modules, cf. [30, Prop. 7.2.4.17]. In particular, $\operatorname{APerf}(R)$ acquires a $t$-structure (by restriction from $\operatorname{Mod}(R)$ ) whose heart is the category of finitely presented discrete $\pi_{0}(R)$-modules; this $t$-structure is right-bounded and left-complete.

\footnotetext{
${ }^{3}$ See also work of Ducros [18] extending the notion of flatness to Berkovich analytic spaces, which we will not consider.
} 
Let $R$ be a coherent $E_{\infty}$-ring. There is similarly a characterization of almost finitely presented $E_{\infty}-R$-algebras. Given any connective $E_{\infty}$-ring $R$, recall [30, Sec. 7.2.4] that a connective $E_{\infty^{-}}$ $R$-algebra $R^{\prime}$ is said to be almost finitely presented if, for each $n$, the truncation $\tau_{\leq n} R^{\prime}$ defines a compact object of the $\infty$-category of connective, $n$-truncated $E_{\infty}-R$-algebras.

Proposition 6.4 (Characterization of almost finitely presented algebras). Let $R$ be a stably coherent $E_{\infty}$-ring. Let $R^{\prime}$ be a connective $E_{\infty}$-R-algebra. Then the following are equivalent:

(1) $R^{\prime}$ is almost finitely presented.

(2) The ring $\pi_{0}\left(R^{\prime}\right)$ is finitely presented as a $\pi_{0}(R)$-algebra. Moreover, $R^{\prime}$ is coherent as an $E_{\infty}$-ring.

In this case, $R^{\prime}$ is stably coherent as well.

Proof. The proof of [30, Prop. 7.2.4.31] (in the noetherian case) works equally here.

Remark 6.5. Coherence will be useful for us in the following situation. Let $R$ be an $E_{\infty}-\mathcal{O}_{K^{-}}$ algebra which is coherent. Suppose that $R[1 / \pi]$ is discrete. Then for each $i>0$, the homotopy group $\pi_{i}(R)$ is $\leq \pi^{\infty}$-isogenous to zero. This follows because, for $i>0, \pi_{i}(R)$ is a finitely presented module over $\pi_{0}(R)$ and $\pi_{i}(R)[1 / \pi]=0$ by assumption.

Example 6.6. The stable coherence of valuation rings (such as $\mathcal{O}_{K}$ ) is a consequence of the results of Raynaud-Gruson [35] (see, e.g., [24, Theorem 7.3.3]). That is, the polynomial ring $\mathcal{O}_{K}\left[T_{1}, \ldots, T_{n}\right]$ is coherent for any $n$. The coherence of the ring $\mathcal{O}_{K}\left\langle T_{1}, \ldots, T_{n}\right\rangle \stackrel{\text { def }}{=} \mathcal{O}_{K}\left[\widehat{T_{1}, \ldots}, T_{n}\right]_{\pi}$ can be deduced similarly; see [12, Sec. 7.3] for an account.

We will need the following strengthening of the above example, which appears in work of Fujiwara-Gabber-Kato [21, Prop. 4.3.4, Prop. 7.2.2, and Th. 7.3.2]. See also 28] for a survey.

Theorem 6.7. Let $K$ be a complete nonarchimedean field. Let $R$ be a finitely presented algebra over the $\pi$-completed polynomial ring $\mathcal{O}_{K}\left\langle T_{1}, \ldots, T_{n}\right\rangle$. Then:

(1) $R$ is coherent.

(2) Given any finitely generated $R$-module $M$, the $\pi$-power torsion submodule is finitely generated. Any $\pi$-torsion-free finitely generated module is finitely presented.

(3) The map from $R$ to its $\pi$-adic completion (which is also the derived $\pi$-adic completion by (2)), $R \rightarrow \hat{R}$, is flat.

In particular, the result implies that for finitely presented algebras over $\mathcal{O}_{K}\left\langle T_{1}, \ldots, T_{n}\right\rangle$ and finitely generated modules, there is no distinction between classical and derived completion.

6.2. The flat topology on $K$-affinoid algebras. Here we review some facts about flatness for maps of $K$-affinoid algebras. For a detailed treatment, see [3, Ch. 5].

Definition 6.8. (1) An $\mathcal{O}_{K}$-algebra $A_{0}$ is topologically of finite presentation if $A_{0}$ is a quotient of some $\mathcal{O}_{K}\left\langle T_{1}, \ldots, T_{n}\right\rangle$ by a finitely generated ideal; if $A_{0}$ is torsion-free, it suffices that $A_{0}$ is a quotient of some $\mathcal{O}_{K}\left\langle T_{1}, \ldots, T_{n}\right\rangle$. Let $\operatorname{Alg}_{\mathcal{O}_{K}}^{\mathrm{tfp}}$ denote the category of $\mathcal{O}_{K}$-algebras which are topologically of finite presentation. We let $\operatorname{Alg}_{\mathcal{O}_{K}}^{\mathrm{tfp}, \mathrm{b}} \subset \operatorname{Alg}_{\mathcal{O}_{K}}^{\mathrm{tfp}}$ be the subcategory of those algebras which are flat (i.e., torsion-free) over $\mathcal{O}_{K}$.

(2) An $K$-affinoid algebra is a $K$-algebra which is a quotient of the Tate algebra $T_{n}=K\left\langle T_{1}, \ldots, T_{n}\right\rangle \stackrel{\text { def }}{=}$ $\left(\mathcal{O}_{K}\left\langle T_{1}, \ldots, T_{n}\right\rangle\right)[1 / \pi]$. Let Affinoid ${ }_{K}$ denote the category of $K$-affinoid algebras. We have an essentially surjective functor $\operatorname{Alg}_{\mathcal{O}_{K}}^{\mathrm{tfp}} \rightarrow$ Affinoid ${ }_{K}$ given by tensoring with $K$. 
The categories $\operatorname{Alg}_{\mathcal{O}_{K}}^{\mathrm{tfp}}$, Affinoid $K$ admit finite colimits via the completed tensor products; the natural functor $\operatorname{Alg}_{\mathcal{O}_{K}}^{\mathrm{tfp}} \rightarrow$ Affinoid $_{K}$ preserves them.

Definition 6.9 (Flat morphisms in $\operatorname{Alg}_{\mathcal{O}_{K}}^{\mathrm{tfp}, b}, \mathrm{Affinoid}_{K}$ ). We define a morphism in $\operatorname{Alg}_{\mathcal{O}_{K}, \mathrm{tp}}^{\mathrm{tp}}$, Affinoid $\mathrm{A}_{K}$ to be flat (resp. faithfully flat) if it is flat (resp. faithfully flat) as a map of ordinary rings. This condition is stable under (completed) base-change:

(1) In $\operatorname{Alg}_{\mathcal{O}_{K}}^{\mathrm{tfp}, b}$, a map $A_{0} \rightarrow B_{0}$ is flat (resp. faithfully flat) if and only if it is flat (resp. faithfully flat) modulo each power of $\pi$. In the case of flatness, this follows because the flatness condition can be tested on coherent (discrete) $A_{0}$-modules, and these are automatically $\pi$-complete. Faithful flatness is implied by flatness and universal descent, so the claim for faithful flatness follows from Lemma 6.15 below.

(2) The statement that flat (resp. faithfully flat) maps in Affinoid $_{K}$ are stable under basechange follows from the existence of flat (resp. faithfully flat) formal models (Theorem 6.11 below).

Example 6.10. (1) Let $A_{0} \rightarrow B_{0}$ be a flat map in $\operatorname{Alg}_{\mathcal{O}_{K}}^{\mathrm{tfp}, b}$. Then $A_{0}[1 / \pi] \rightarrow B_{0}[1 / \pi]$ is a flat map of $K$-affinoid algebras.

(2) Let $A_{0} \in \operatorname{Alg}_{\mathcal{O}_{K}}^{\mathrm{tfp}}$ and $A_{0}^{\prime}$ be a finitely presented $A_{0}$-algebra. Suppose $A_{0}[1 / \pi] \rightarrow A_{0}^{\prime}[1 / \pi]$ is flat. Then the map $A_{0}[1 / \pi] \rightarrow \widehat{A_{0}^{\prime}}[1 / \pi]$ of $K$-affinoid algebras is flat. Indeed, this follows because $A_{0}^{\prime} \rightarrow \widehat{A_{0}^{\prime}}$ is flat (Theorem 6.7).

One can ask when a map in $\operatorname{Alg}_{\mathcal{O}_{K}}^{\mathrm{tfp}, b}$ has flat generic fiber. It turns out that the above two are the essential cases. To see this, we will use the flattening results of [14], after the work of RaynaudGruson [35] in the case of schemes. See also [3, Sec. 5.8] for an account. The last assertion (of faithful flatness rather than flatness) follows from [3, Prop. 5.5.10].

Theorem 6.11 (Bosch-Lütkebohmert [14]). Let $A_{0} \rightarrow B_{0}$ be a map in $\mathrm{Alg}_{\mathcal{O}_{K}}^{\mathrm{tfp}, b}$. Let $\mathfrak{X}=\operatorname{Spf} A_{0}, \mathfrak{Y}=$ Spf $B_{0}$ and let $f: \mathfrak{Y} \rightarrow \mathfrak{X}$ be the induced map. Suppose the map $A_{0}[1 / \pi] \rightarrow B_{0}[1 / \pi]$ is flat. There exists a diagram of formal schemes

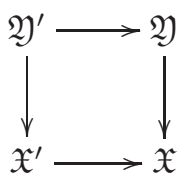

such that:

(1) $\mathfrak{X}^{\prime} \rightarrow \mathfrak{X}$ is a formal blow-up along an open, finitely generated ideal $I \subset A_{0}$.

(2) $\mathfrak{Y}^{\prime}$ is the strict transform of $\mathfrak{Y}$.

(3) $\mathfrak{Y}^{\prime} \rightarrow \mathfrak{X}^{\prime}$ is flat.

Moreover, if $A_{0}[1 / \pi] \rightarrow B_{0}[1 / \pi]$ is faithfully flat, then $\mathfrak{Y}^{\prime} \rightarrow \mathfrak{X}^{\prime}$ is also faithfully flat.

We can restate the above result purely in terms of schemes (rather than formal schemes), and in the faithfully flat case, as follows.

Proposition 6.12. Let $A_{0} \rightarrow B_{0}$ be a map in $\operatorname{Alg}_{\mathcal{O}_{K}}^{\mathrm{tfp}, b}$ such that $A_{0}[1 / \pi] \rightarrow B_{0}[1 / \pi]$ is faithfully flat. Then there exists a finitely presented $A_{0}$-algebra $A_{0}^{\prime}$, flat over $\mathcal{O}_{K}$, such that:

(1) $A_{0} \rightarrow A_{0}^{\prime}$ is a universal descent morphism.

(2) $A_{0}[1 / \pi] \rightarrow A_{0}^{\prime}[1 / \pi]$ is faithfully flat. 
(3) The map $A_{0}^{\prime} \rightarrow B_{0}^{\prime} \stackrel{\text { def }}{=} \operatorname{Tor}_{0}^{A_{0}}\left(B_{0}, A_{0}^{\prime}\right)=\pi_{0}\left(B_{0} \otimes_{A_{0}} A_{0}^{\prime}\right)$ has the property that $A_{0}^{\prime} \rightarrow$ $B_{0}^{\prime} /\left\{\pi^{\infty}\right.$ - torsion $\}$ is faithfully flat.

Proof. This follows from Theorem 6.11 instead of taking the formal blow-up along the relevant (finitely generated) ideal $I \subset A_{0}$, we take the actual scheme-theoretic blowup $X^{\prime} \rightarrow X=\operatorname{Spec}\left(A_{0}\right)$; note that $X^{\prime}[1 / \pi] \rightarrow X[1 / \pi]$ is an isomorphism. Since $I$ is finitely generated, $X^{\prime} \rightarrow X$ is a finite type map of schemes; since everything is flat over $\mathcal{O}_{K}, X^{\prime} \rightarrow X$ is finitely presented (Theorem 6.7). If we let $Y^{\prime}$ be the strict transform of $Y=\operatorname{Spec}\left(B_{0}\right)$, then the previous result states that $Y^{\prime} \rightarrow X^{\prime}$ defines a map of $\mathcal{O}_{K}$-schemes which is flat modulo $\pi$. We can then take $A_{0}^{\prime}$ to be an affine cover of $X^{\prime}$.

Note that $A_{0}[1 / \pi] \rightarrow A_{0}^{\prime}[1 / \pi]$ is a Zariski cover, verifying (2). Letting $B_{0}^{\prime b}=B_{0}^{\prime} /\left\{\pi^{\infty}-\right.$ torsion $\}$, the assertions of Theorem 6.11 give that $A_{0}^{\prime} \rightarrow B_{0}^{\prime b}$ becomes faithfully flat modulo any power of $\pi$. Moreover, it is faithfully flat after inverting $\pi$ by base-change, by our assumptions. This implies that $A_{0}^{\prime} \rightarrow B_{0}^{\prime b}$ is faithfully flat. Finally, to see that $A_{0} \rightarrow A_{0}^{\prime}$ is a universal descent morphism, we argue via Theorem 5.5 indeed, $X^{\prime} \rightarrow X$ is a proper, finitely presented and surjective morphism and hence a $v$-cover. It follows that $A_{0} \rightarrow A_{0}^{\prime}$ is a finitely presented $v$-cover and hence a universal descent morphism.

Lemma 6.13 (Discreteness up to quasi-isogeny criterion). Let $A_{0} \in \operatorname{Alg}_{\mathcal{O}_{K}}^{\mathrm{tfp}}$. Let $A_{0}^{\prime}$ be a finitely presented $A_{0}$-algebra such that:

(1) $A_{0} \rightarrow A_{0}^{\prime}$ is a universal descent morphism.

(2) The map $A_{0}[1 / \pi] \rightarrow A_{0}^{\prime}[1 / \pi]$ is flat.

Let $M \in \operatorname{Mod}\left(A_{0}\right)_{\geq 0}$. Suppose the (derived) base-change $M \otimes_{A_{0}} A_{0}^{\prime} \in \operatorname{Mod}\left(A_{0}^{\prime}\right)$ is quasi-isogenous to a flat, discrete $A_{0}^{\prime}$-module. Then each (derived) tensor power $M^{\otimes n} \in \operatorname{Mod}\left(A_{0}\right), n \geq 0$ is quasiisogenous to a discrete module.

Proof. Our assumptions imply that each $M^{\otimes n} \otimes_{A_{0}}\left(A_{0}^{\prime} \otimes_{A_{0}} \cdots \otimes_{A_{0}} A_{0}^{\prime}\right)$ is quasi-isogenous to a flat $A_{0}^{\prime} \otimes_{A_{0}} \cdots \otimes_{A_{0}} A_{0}^{\prime}$-module. Now $A_{0} \rightarrow A_{0}^{\prime}$ is finitely presented as a map of rings, and hence almost finitely presented as a map of $E_{\infty}$-rings (by Proposition 6.4 and Theorem 6.7). Therefore, the iterated tensor products $A_{0}^{\prime} \otimes_{A_{0}} \cdots \otimes_{A_{0}} A_{0}^{\prime}$ (as $E_{\infty}$-algebras over $A_{0}$ ) are almost finitely presented $E_{\infty}$-algebras over $A_{0}$ and become discrete after inverting $\pi$, by (2). Therefore, their higher homotopy groups are all $\leq \pi^{\infty}$-isogenous to zero by coherence (cf. Remark 6.5). In particular, we find that each $M^{\otimes n} \otimes_{A_{0}}\left(A_{0}^{\prime} \otimes_{A_{0}} \cdots \otimes_{A_{0}} A_{0}^{\prime}\right)$ is quasi-isogenous to a discrete module. Using the canonical resolution of $M^{\otimes n}$ by its base-changes to the iterative tensor powers $A_{0}^{\prime} \otimes_{A_{0}} \otimes \cdots \otimes_{A_{0}} A_{0}^{\prime}$ and taking some $\operatorname{Tot}^{N}$ (since $A_{0} \rightarrow A_{0}^{\prime}$ is universal descent), we can conclude the result.

Proposition 6.14. Let $A_{0} \rightarrow B_{0}$ be a map in $\operatorname{Alg}_{\mathcal{O}_{K}}^{\mathrm{tfp}, \mathrm{b}}$. Suppose the map $A_{0}[1 / \pi] \rightarrow B_{0}[1 / \pi]$ in Affinoid $_{K}$ is faithfully flat. Then:

(1) $A_{0} \rightarrow B_{0}$ is a universal descent morphism.

(2) For each $n$, the derived tensor product $B_{0} \otimes_{A_{0}} \cdots \otimes_{A_{0}} B_{0} \in \operatorname{Mod}\left(A_{0}\right)$ has bounded $\pi$-power torsion in each homological degree, and is all $\pi$-torsion in positive degrees.

Proof. By Proposition 6.12, there exists a finitely presented, universal descent, discrete $A_{0}$-algebra $A_{0}^{\prime}$ such that $A_{0}[1 / \pi] \rightarrow A_{0}^{\prime}[1 / \pi]$ is flat and such that $B_{0}^{\prime b} \stackrel{\text { def }}{=} \pi_{0}\left(B_{0} \otimes_{A_{0}} A_{0}^{\prime}\right) /\left\{\pi^{\infty}-\right.$ torsion $\}$ is faithfully flat over $A_{0}^{\prime}$. Then the (derived) tensor product $B_{0} \otimes_{A_{0}} A_{0}^{\prime}$ has the structure of an almost finitely presented $E_{\infty}-B_{0}$-algebra which becomes discrete after inverting $\pi$. By coherence (Theorem 6.7 and Remark 6.5), $B_{0} \otimes_{A_{0}} A_{0}^{\prime}$ is quasi-isogenous to the flat, discrete $A_{0}^{\prime}$-module $B_{0}^{\prime \prime}$ : 
in particular, the higher homotopy groups and the torsion in $\pi_{0}$ are bounded torsion. The claim (2) now follows from Lemma 6.13.

Finally, we need to verify that $A_{0} \rightarrow B_{0}$ is universal descent. By assumption, $A_{0} \rightarrow A_{0}^{\prime}$ is a universal descent morphism. Since the source is $\pi$-complete, it follows that $A_{0} \rightarrow \widehat{A_{0}^{\prime}}$ is a universal descent morphism (Lemma 6.15). Therefore, it suffices to show (by two-out-of-three as in Remark [5.2) that $\widehat{A_{0}^{\prime}} \rightarrow \widehat{B_{0}^{\prime \prime}}$ is a universal descent morphism: indeed, then $A_{0} \rightarrow \widehat{B_{0}^{\prime \prime}}$ is universal descent and hence so is $A_{0} \rightarrow B_{0}$. But $\widehat{A_{0}^{\prime}} \rightarrow \widehat{B_{0}^{\prime \prime}}$ is faithfully flat and finitely presented modulo $\pi$, so the claim again follows from Lemma 6.15 .

Lemma 6.15. Let $R \rightarrow S$ be a map of $\pi$-torsion-free $\mathcal{O}_{K}$-algebras. Suppose $R$ is $\pi$-adically complete. Then:

(1) $R \rightarrow S$ is a universal descent morphism if and only if $R \rightarrow \widehat{S}$ is a universal descent morphism.

(2) Suppose $R / \pi \rightarrow S / \pi$ is faithfully flat and finitely presented. Then $R \rightarrow S$ is a universal descent morphism.

Proof. Let $I=\operatorname{fib}(R \rightarrow S)$ in $\operatorname{Mod}(R)$, so we have a canonical map $f: I \rightarrow R$. Then $R \rightarrow S$ is a universal descent morphism if and only if $f^{\otimes n}: I^{\otimes n} \rightarrow R$ is nullhomotopic (in $\left.\operatorname{Mod}(R)\right)$ for $n \gg 0$. Similarly, $R \rightarrow \widehat{S}$ is universal descent if and only if $(\widehat{I})^{\otimes n} \rightarrow R$ is nullhomotopic for $n \gg 0$. These conditions are equivalent because $I^{\otimes n},(\widehat{I})^{\otimes n}$ have the same $\pi$-completion and $R$ is $\pi$-complete. This proves (1).

Now suppose $R / \pi \rightarrow S / \pi$ is faithfully flat and finitely presented. By [31, Lemma D.3.3.7], the map $f^{\otimes n}$ vanishes after base-change to any quotient $\mathcal{O}_{K} / \pi^{k}$ for $n \geq 2$; indeed, $I^{\otimes n} / \pi^{k}$ is the $(-n)$-shift of a flat discrete $R / \pi^{k}$-module which is at most countably presented, whence $\pi_{0} \operatorname{Hom}_{R / \pi^{k}}\left(I^{\otimes n} / \pi^{k}, R / \pi^{k}\right)=0$ by loc. cit.. Therefore, the map $f^{\otimes 2}: \widehat{I^{\otimes 2}} \rightarrow R$ is divisible by any power of $\pi$. Consider the composable maps $\pi_{0} \operatorname{Hom}_{R}\left(\widehat{I^{\otimes 4}}, \widehat{I^{\otimes 4}}\right) \stackrel{f^{\otimes 2} \circ}{\longrightarrow} \pi_{0} \operatorname{Hom}_{R}\left(\widehat{I^{\otimes 4}}, \widehat{I^{\otimes 2}}\right) \stackrel{f^{\otimes 2} \circ}{\longrightarrow}$ $\pi_{0} \operatorname{Hom}_{R}\left(\widehat{I^{\otimes 4}}, R\right)$, each of which is divisible by any power of $\pi$; it follows from Lemma 2.28 that the composite is zero. Therefore, $f^{\otimes 4}$ is nullhomotopic as desired.

Theorem 6.16. The construction $A \mapsto \operatorname{APerf}(A)$ defines a hypercomplete sheaf of $\infty$-categories for the flat topology on $\left(\text { Affinoid }_{K}\right)^{o p}$.

Proof. Let $A \rightarrow B$ be a faithfully flat map in Affinoid $_{K}$. Choose open subrings $A_{0} \subset A, B_{0} \subset B$ in $\operatorname{Alg}_{\mathcal{O}_{K}}^{\mathrm{tfp}, b}$ such that $A_{0}$ is carried to $B_{0}$. Then Čech descent of $\operatorname{APerf}(\cdot)$ along $A \rightarrow B$ follows from Proposition 6.14 and Theorem [5.8. From this, it follows by flatness that the subcategories $\operatorname{APerf}(\cdot)_{[a, b]}$ form sheaves on on Affinoid $_{K}$ for each $a \leq b$; these sheaves are necessarily hypercomplete since they take values in truncated $\infty$-categories. Taking the limit in $a, b$, we find that $\operatorname{APerf}(\cdot)$ is a hypercomplete sheaf.

\section{Flat DESCEnt}

In this section, we prove our main faithfully flat descent theorem (Theorem 7.8). Let $R$ be a connective $E_{\infty}$-ring with a finitely generated ideal $I \subset \pi_{0}(R)$. We need the following definition, which generalizes Definition 1.5. For an $E_{\infty}$-R-algebra $S$, we denote by $\operatorname{Mod}(S)_{I-\text { tors }}$ the full subcategory of $\operatorname{Mod}(S)$ spanned by the $I$-torsion modules. 
Definition 7.1 ( $I$-complete flatness, after [9, Sec. 4] and [11, Notations]). Let $S \rightarrow S^{\prime}$ be a map of connective $E_{\infty}$-rings under $R$. We will say that $S \rightarrow S^{\prime}$ is I-completely flat (resp. I-completely faithfully flat) if the base-change functor $\operatorname{Mod}(S)_{I \text {-tors }} \rightarrow \operatorname{Mod}\left(S^{\prime}\right)_{I \text {-tors }}$ is $t$-exact (resp. $t$-exact and conservative). For instance, if $S \rightarrow S^{\prime}$ is map of discrete rings and $I \subset R$ is generated by a nonzerodivisor $u$, then this holds if $u$ is a nonzerodivisor in $S, S^{\prime}$ and $S / u \rightarrow S^{\prime} / u$ is flat (resp. faithfully flat). This defines the $I$-completely flat topology on the opposite of the $\infty$-category of connective $E_{\infty}$ - $R$-algebras.

Remark 7.2. Let $S \rightarrow S^{\prime}$ be $I$-completely faithfully flat. Let $M \in \operatorname{Mod}(S)_{I-\text { tors }}$. Then for each $i$ and each $r \geq 0$, the following are equivalent:

(1) $\pi_{i}(M)$ is annihilated by $I^{r}$.

(2) $\pi_{i}\left(S^{\prime} \otimes_{S} M\right)$ is annihilated by $I^{r}$.

This follows since base-change is $t$-exact and conservative.

Proposition 7.3 (Flat hyperdescent for $\mathcal{M}(R)$ ). The construction $S \mapsto \mathcal{M}(S)$ defines a hypercomplete sheaf for the I-completely flat topology. Similarly for $S \mapsto \mathcal{M}(S)_{\geq 0}$.

Proof. This follows from Proposition 4.6. Indeed, if $S \rightarrow S^{\prime}$ is $I$-completely faithfully flat, then the functor $\mathcal{M}(S) \rightarrow \mathcal{M}\left(S^{\prime}\right)$ of stable $\infty$-categories equipped with right-bounded, left-complete $t$-structures (specifically, the $I$-torsion $t$-structures) is conservative and $t$-exact. Therefore, we find that for each $m \leq n, S \mapsto \mathcal{M}(S)_{[m, n]}$ is a sheaf for the $I$-completely flat topology (necessarily hypercomplete since these are truncated $\infty$-categories); taking the inverse limit over $n$ and then the direct limit over $m$, we find that $S \mapsto \mathcal{M}(S)$ is a hypercomplete sheaf. Similarly for $S \mapsto$ $\mathcal{M}(S)_{\geq 0}$.

Proposition 7.4. Suppose $S \rightarrow S^{\prime}$ is an I-completely faithfully flat map of connective $E_{\infty}-R$ algebras. Let $M \in \operatorname{Mod}(S)_{>-\infty}^{\mathrm{cpl}}$. Then the following are equivalent:

(1) $\pi_{i}(M)$ is $\leq I^{\infty}$-isogenous to zero for $i<0$ (i.e., $M$ defines a connective object of $\mathcal{M}_{0}(S)$ ).

(2) $\pi_{i}\left(\widehat{M \otimes_{S}} S^{\prime}\right)$ is $\leq I^{\infty}$-isogenous to zero for $i<0$ (i.e., $\widehat{M \otimes_{S}} S^{\prime}$ defines a connective object of $\mathcal{M}_{0}\left(S^{\prime}\right)$ ).

Proof. Fix a tower $\left\{S_{n}\right\}$ as in Construction 2.32, By Proposition 2.34, the first condition is equivalent to the assertion that for each $i<0$, the modules $\pi_{i}\left(M \otimes_{S} S_{n}\right)$ are $\leq I^{r_{1}}$-isogenous to zero for some $r_{1}$ and all $n$. Similarly, the second condition is equivalent to the assertion that for each $i<0$, the modules $\pi_{i}\left(\left(M \otimes_{S} S_{n}\right) \otimes_{S} S^{\prime}\right)$ are $\leq I^{r_{2}}$-isogenous to zero for some $r_{2}$ and all $n$. But these two conditions are then equivalent as in Remark 7.2 .

Proposition 7.5. Let $S \rightarrow S^{\prime}$ be an I-completely faithfully flat map of connective $E_{\infty}$-R-algebras. Let $M \in \operatorname{Mod}(S)_{I-\text { tors }}$ be connective. Then for any $S$, the following are equivalent:

(1) $M$ is $\leq I^{r}$-perfect to order zero as an $S$-module.

(2) $S^{\prime} \otimes_{S} M$ is $\leq I^{r}$-perfect to order zero as an $S^{\prime}$-module.

Proof. Clearly (1) implies (2). Suppose (2). Fix a tower $\left\{S_{n}\right\}$ as in Construction 2.32. Then there exists a map $f_{1}: S^{\prime m} \rightarrow S^{\prime} \otimes_{S} M$ whose cofiber has $\pi_{0}$ annihilated by $I^{r}$. Up to increasing $m$, we can assume $f_{1}$ is the base-change of a map $f_{0}: S^{m} \rightarrow M$. This factors over a map $f_{0}: S_{n}^{m} \rightarrow M$ for $n \gg 0$, since $M$ is torsion. It now follows by descent (Remark 7.2) that $\pi_{0}\left(\operatorname{cofib}\left(f_{0}\right)\right)$ is annihilated by $\leq I^{r}$. Therefore, $M$ is $\leq I^{r}$-perfect to order zero.

Proposition 7.6. Suppose $S \rightarrow S^{\prime}$ is an I-completely faithfully flat map of I-complete connective $E_{\infty}-R$-algebras. Let $M \in \operatorname{Mod}(S)_{>-\infty}^{\mathrm{cpl}}$. Then the following are equivalent: 
(1) $M$ is $\leq I^{\infty}$-perfect to order $n$.

(2) $\widehat{M \otimes_{S}} S^{\prime}$ is $\leq I^{\infty}$-perfect to order $n$.

Proof. Without loss of generality, we can assume that $M$ is actually connective as an $R$-module. It suffices to show that (2) implies (1). We use induction on $n$. Suppose $n=0$. Fix a tower $\left\{S_{m}\right\}$ as in Construction 2.32. By Proposition 2.30, the first condition is equivalent to the assertion that there exists some $r_{1}$ such that $M \otimes_{S} S_{m}$ is $\leq I^{r_{1}}$-perfect to order zero for all $m$. Similarly, the second condition is equivalent to the assertion that there exists some $r_{2}$ such that $M \otimes_{S} S_{m} \otimes_{S} S^{\prime}$ is $\leq I^{r_{2}}$ perfect to order zero for all $m$. The two conditions are thus equivalent thanks to Proposition 7.5

Suppose (2) with $n>0$. From what we have already shown, $\pi_{0}(M)$ is $\leq I^{\infty}$-finitely generated, and up to modifying $M$ up to $\leq I^{\infty}$-isogeny we may assume that $\pi_{0}(M)$ is finitely generated. Replacing $M$ with the fiber of a map $S^{r} \rightarrow M$ inducing a surjection on $\pi_{0}$ and using Proposition 2.26 (as well as the inductive step), we can now conclude (1) for $M$.

Proposition 7.7. Suppose $S \rightarrow S^{\prime}$ is an I-completely faithfully flat map of I-complete connective $E_{\infty}-R$-algebras. Let $M \in \mathcal{M}(S)$. Then the following are equivalent:

(1) $M$ is weakly perfect to order $n$.

(2) The base-change $S^{\prime} \otimes_{S} M \in \mathcal{M}\left(S^{\prime}\right)$ is weakly perfect to order $n$.

Proof. Both of these conditions only depend on $\tau_{\leq n} M$, so we can assume $M \in \mathcal{M}(S)_{\leq n}=\mathcal{M}_{0}(S)_{\leq n}$ and represent $M$ by an object $M^{\prime}$ of $\operatorname{Mod}(S)_{>-\infty}^{\mathrm{cpl}}$. Then condition (1) is equivalent to the assertion that $M^{\prime}$ is $\leq I^{\infty}$-perfect to order $n$ while condition (2) is equivalent to the assertion that $\overline{M \otimes_{S}} S^{\prime}$ is $\leq I^{\infty}$-perfect to order $n$. The result follows from Proposition 7.6 .

Now we can prove the main descent theorem of this article, which generalizes the results of Drinfeld [16, Th. 3.11] and [17, Prop. 3.5.4]. Heuristically, Theorem 7.8 suggests that almost perfect complexes (defined purely algebraically) behave well in analytic geometry quite generally. Closely related is Kedlaya-Liu [29, Sec. 2] which constructs a category of pseudocoherent sheaves on adic spaces (corresponding to almost perfect complexes which are discrete). See also HennionPorta-Vezzosi 25] for the case of algebras finite type over a field with the étale topology, and generalizations to stacks. We refer to 38 for a new approach to defining analogs of "big" categories of quasi-coherent sheaves in analytic geometry, from which we expect it should be possible to recover our results 4

Theorem 7.8 (Faithfully flat descent for APerf). The construction $S \mapsto \operatorname{APerf}\left(\operatorname{Spec}\left(\hat{S}_{I}\right) \backslash V(I)\right)$ defines a hypercomplete sheaf for the I-completely flat topology. Similarly for the subcategories APerf $_{\geq 0}$ of connective almost perfect modules, Perf of perfect modules, and $\operatorname{Perf}_{[a, b]}$ of perfect modules with Tor-amplitude in $[a, b]$ for any $a \leq b$.

Proof. First, we have seen that $S \mapsto \mathcal{M}(S)$ is a hypercomplete sheaf of $\infty$-categories (Proposition (7.3). Second, there is an embedding $\operatorname{APerf}(\operatorname{Spec}(S) \backslash V(I)) \subset \mathcal{M}(S)$ (whose inverse is given by the functor $j^{*}$ ), by Proposition 3.16. Third, the condition of belonging to APerf is local (Proposition (7.7). Combining these three assertions, we find that $S \mapsto \operatorname{APerf}\left(\operatorname{Spec}\left(\hat{S}_{I}\right) \backslash V(I)\right)$ is a hypercomplete sheaf.

We can carry out a similar argument for $\operatorname{APerf}(\cdot)_{\geq 0}$, since we also know that $S \mapsto \mathcal{M}(S)_{\geq 0}$ is a hypercomplete sheaf as well and $\operatorname{APerf}\left(\operatorname{Spec}\left(\widehat{S}_{I}\right) \backslash V(I)\right)_{>0} \subset \mathcal{M}(S)_{>0}$ (as in loc. cit.). The result for APerf implies the result for Perf by taking the subcategories of dualizable objects. The result

\footnotetext{
${ }^{4}$ Compare the recent work of Andreychev, 4].
} 
for Perf $\geq 0$ now follows by taking the intersection of APerf $\geq 0$ and Perf. Finally, $\operatorname{Perf}_{[a, b]}$ is given by those objects of Perf which belong to $\operatorname{Perf}_{\geq a}$ and whose dual belongs to Perf $\geq-b$, so this is also a local condition.

\section{REFERENCES}

[1] Théorie des intersections et théorème de Riemann-Roch. Lecture Notes in Mathematics, Vol. 225. SpringerVerlag, Berlin-New York, 1971. Séminaire de Géométrie Algébrique du Bois-Marie 1966-1967 (SGA 6), Dirigé par P. Berthelot, A. Grothendieck et L. Illusie. Avec la collaboration de D. Ferrand, J. P. Jouanolou, O. Jussila, S. Kleiman, M. Raynaud et J. P. Serre.

[2] Revêtements étales et groupe fondamental (SGA 1), volume 3 of Documents Mathématiques (Paris) [Mathematical Documents (Paris)]. Société Mathématique de France, Paris, 2003. Séminaire de géométrie algébrique du Bois Marie 1960-61. [Algebraic Geometry Seminar of Bois Marie 1960-61], Directed by A. Grothendieck, With two papers by M. Raynaud, Updated and annotated reprint of the 1971 original [Lecture Notes in Math., 224, Springer, Berlin; MR0354651 (50 \#7129)].

[3] Ahmed Abbes. Éléments de géométrie rigide. Volume I, volume 286 of Progress in Mathematics. Birkhäuser/Springer Basel AG, Basel, 2010. Construction et étude géométrique des espaces rigides. [Construction and geometric study of rigid spaces], With a preface by Michel Raynaud.

[4] Grigory Andreychev. Pseudocoherent and perfect complexes and vector bundles on analytic adic spaces. arXiv preprint arXiv:2105.12591, 2021.

[5] Tobias Barthel and A. K. Bousfield. On the comparison of stable and unstable p-completion. Proc. Amer. Math. Soc., 147(2):897-908, 2019.

[6] A. A. Beulinson, J. Bernstein, and P. Deligne. Faisceaux pervers. In Analysis and topology on singular spaces, I (Luminy, 1981), volume 100 of Astérisque, pages 5-171. Soc. Math. France, Paris, 1982.

[7] Bhargav Bhatt. Torsion completions are bounded. J. Pure Appl. Algebra, 223(5):1940-1945, 2019.

[8] Bhargav Bhatt and Akhil Mathew. The arc-topology. Duke Math. J., 170(9):1899-1988, 2021.

[9] Bhargav Bhatt, Matthew Morrow, and Peter Scholze. Topological Hochschild homology and integral $p$-adic Hodge theory. Publ. Math. Inst. Hautes Études Sci., 129:199-310, 2019.

[10] Bhargav Bhatt and Peter Scholze. The pro-étale topology for schemes. Astérisque, (369):99-201, 2015.

[11] Bhargav Bhatt and Peter Scholze. Prisms and prismatic cohomology. arXiv preprint arXiv:1905.08229, 2019.

[12] Siegfried Bosch. Lectures on formal and rigid geometry, volume 2105 of Lecture Notes in Mathematics. Springer, Cham, 2014.

[13] Siegfried Bosch and Ulrich Görtz. Coherent modules and their descent on relative rigid spaces. J. Reine Angew. Math., 495:119-134, 1998.

[14] Siegfried Bosch and Werner Lütkebohmert. Formal and rigid geometry. II. Flattening techniques. Math. Ann., 296(3):403-429, 1993.

[15] Brian Conrad. Descent for coherent sheaves on rigid analytic spaces. Available at http://math.stanford.edu/ conrad/papers/cohdescent.pdf.

[16] Vladimir Drinfeld. Infinite-dimensional vector bundles in algebraic geometry: an introduction. In The unity of mathematics, volume 244 of Progr. Math., pages 263-304. Birkhäuser Boston, Boston, MA, 2006.

[17] Vladimir Drinfeld. A stacky approach to crystals. 2018. arXiv:1810.11853.

[18] Antoine Ducros. Flatness in non-Archimedean analytic geometry. Available at https://webusers.imj-prg.fr/ antoine.ducros/Platitude.pdf

[19] W. G. Dwyer and J. P. C. Greenlees. Complete modules and torsion modules. Amer. J. Math., 124(1):199-220, 2002.

[20] Renée Elkik. Solutions d'équations à coefficients dans un anneau hensélien. Ann. Sci. École Norm. Sup. (4), 6:553-603 (1974), 1973.

[21] Kazuhiro Fujiwara, Ofer Gabber, and Fumiharu Kato. On Hausdorff completions of commutative rings in rigid geometry. J. Algebra, 332:293-321, 2011.

[22] Ofer Gabber. Affine analog of the proper base change theorem. Israel J. Math., 87(1-3):325-335, 1994.

[23] Ofer Gabber and Lorenzo Ramero. Almost ring theory, volume 1800 of Lecture Notes in Mathematics. SpringerVerlag, Berlin, 2003.

[24] Sarah Glaz. Commutative coherent rings, volume 1371 of Lecture Notes in Mathematics. Springer-Verlag, Berlin, 1989. 
[25] Benjamin Hennion, Mauro Porta, and Gabriele Vezzosi. Formal gluing along non-linear flags, 2016. arXiv:1607.04503.

[26] Mark Hovey, John H. Palmieri, and Neil P. Strickland. Axiomatic stable homotopy theory. Mem. Amer. Math. Soc., 128(610):x+114, 1997.

[27] Luc Illusie, Yves Laszlo, and Fabrice Orgogozo, editors. Travaux de Gabber sur l'uniformisation locale et la cohomologie étale des schémas quasi-excellents. Société Mathématique de France, Paris, 2014. Séminaire à l'École Polytechnique 2006-2008. [Seminar of the Polytechnic School 2006-2008], With the collaboration of Frédéric Déglise, Alban Moreau, Vincent Pilloni, Michel Raynaud, Joël Riou, Benoît Stroh, Michael Temkin and Weizhe Zheng, Astérisque No. 363-364 (2014) (2014).

[28] Fumiharu Kato. Topological rings in rigid geometry. In Motivic integration and its interactions with model theory and non-Archimedean geometry. Volume I, volume 383 of London Math. Soc. Lecture Note Ser., pages 103-144. Cambridge Univ. Press, Cambridge, 2011.

[29] Kiran S. Kedlaya and Ruochuan Liu. Relative p-adic Hodge theory, II: Imperfect period rings. 2016. arXiv:1602.06899.

[30] Jacob Lurie. Higher algebra. Available at https://www.math.ias.edu/ lurie/papers/HA.pdf

[31] Jacob Lurie. Spectral algebraic geometry. Available at https://www.math.ias.edu/ lurie/papers/SAG-rootfile.pdf

[32] Akhil Mathew. The Galois group of a stable homotopy theory. Adv. Math., 291:403-541, 2016.

[33] Akhil Mathew. Examples of descent up to nilpotence. In Geometric and topological aspects of the representation theory of finite groups, volume 242 of Springer Proc. Math. Stat., pages 269-311. Springer, Cham, 2018.

[34] Arthur Ogus. F-isocrystals and de Rham cohomology. II. Convergent isocrystals. Duke Math. J., 51(4):765-850, 1984.

[35] Michel Raynaud and Laurent Gruson. Critères de platitude et de projectivité. Techniques de "platification" d'un module. Invent. Math., 13:1-89, 1971.

[36] John Rognes. Galois extensions of structured ring spectra. Stably dualizable groups. Mem. Amer. Math. Soc., 192(898):viii+137, 2008.

[37] David Rydh. Submersions and effective descent of étale morphisms. Bull. Soc. Math. France, 138(2):181-230, 2010.

[38] Peter Scholze. Lectures on analytic geometry. Available at https://www.math.uni-bonn.de/people/scholze/Analytic.pdf

[39] Peter Scholze. Étale cohomology of diamonds. 2017. arXiv:1709.07343.

[40] The Stacks project authors. The Stacks Project. https://stacks.math.columbia.edu, 2019.

[41] R. W. Thomason. The classification of triangulated subcategories. Compositio Math., 105(1):1-27, 1997. 